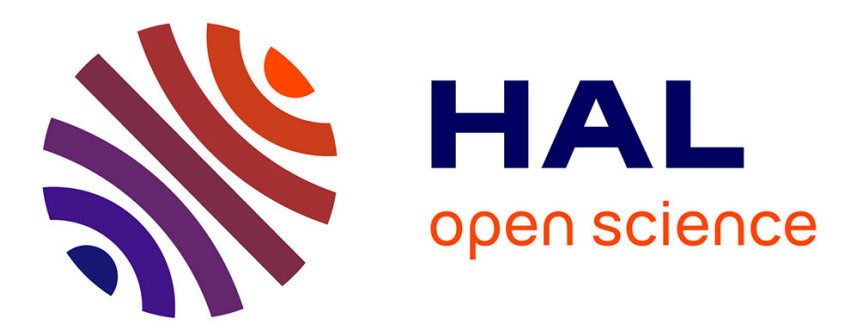

\title{
Comparison of passive damping treatments based on constrained viscoelastic layers and multi-resonant piezoelectric networks
}

\author{
Boris Lossouarn, Lucie Rouleau, Robin Darleux, Jean-François Deü
}

\section{To cite this version:}

Boris Lossouarn, Lucie Rouleau, Robin Darleux, Jean-François Deü. Comparison of passive damping treatments based on constrained viscoelastic layers and multi-resonant piezoelectric networks. Journal of Structural Dynamics, 2021, 10.25518/2684-6500.63 . hal-03445574

\author{
HAL Id: hal-03445574 \\ https://hal.science/hal-03445574
}

Submitted on 25 Nov 2021

HAL is a multi-disciplinary open access archive for the deposit and dissemination of scientific research documents, whether they are published or not. The documents may come from teaching and research institutions in France or abroad, or from public or private research centers.
L'archive ouverte pluridisciplinaire HAL, est destinée au dépôt et à la diffusion de documents scientifiques de niveau recherche, publiés ou non, émanant des établissements d'enseignement et de recherche français ou étrangers, des laboratoires publics ou privés. 


\title{
Comparison of passive damping treatments based on constrained viscoelastic layers and multi-resonant piezoelectric networks
}

\author{
Boris Lossouarn; Lucie Rouleau, Robin Darleux, and Jean-François Deü ${ }^{\ddagger}$ \\ Laboratoire de Mécanique des Structures et des Systèmes Couplés, Conservatoire national des arts et métiers, 292 Rue \\ Saint-Martin, 75003 Paris, France
}

\begin{abstract}
This work aims at comparing the damping performances of two passive damping treatments based on piezoelectric or viscoelastic patches. The motivation for such a comparison stems from the fact that the two damping techniques have been developed fairly independently, and are rarely compared. First, the dynamic response of a simply-supported metallic plate is measured experimentally after being equipped with constrained viscoelastic patches or piezoelectric patches connected to an electrical network. In order to extend the comparison, a numerical model of the structure is set up and validated to evaluate the damping performances of both passive treatments under different configurations (for instance equal-mass and equal-thickness configurations). Finally, with regard to these experimental and numerical results, the advantages and the limitations in using viscoelastic or piezoelectric treatments are discussed.
\end{abstract}

Keywords: Broadband vibration mitigation; Passive vibration control; Piezoelectric damping; Viscoelastic damping

\section{Introduction}

In the context of noise and vibration mitigation, several damping technologies are proposed in the literature. Among them, purely passive treatments generally lead to low-cost and robust structural vibration control. These can be achieved by bonding constrained viscoelastic patches to the structure or by using piezoelectric patches connected to an electrical network. With viscoelastic patches, the vibrational energy is transferred to the viscoelastic layer undergoing shear deformations and is dissipated in the damping material [1, 2]. On the other hand, with piezoelectric patches, the vibrational energy is transferred to an electrical circuit and dissipated in resistive components [3]. Resonant piezoelectric shunts are commonly tuned to a single mechanical natural frequency to be controlled [4, 5, 6, 7, 8]. However, broadband vibration reduction can also be obtained with piezoelectric multimodal damping, where the structure is coupled to a multi-resonant electrical network [9, 10, 11, 12, 13]. On the other hand, viscoelastic patches naturally provide broadband damping [14].

Both passive damping treatments have been developed fairly independently and are rarely compared [15, 16, 17]. The goal of this work, initiated in [16], is to provide an experimental and a numerical comparison of the damping performance of constrained viscoelastic layers and passive piezoelectric networks. In this study, we consider a simply-supported metallic plate, equipped with constrained viscoelastic patches or piezoelectric patches connected to a multi-resonant electrical network.

The next section describes the original experimental setup and reports the results obtained using the piezoelectric and viscoelastic panels that represent the starting point of the present study. The efficiency of both passive treatments usually depends on the volume of material used, so that the performance is generally limited by weight and size

\footnotetext{
*boris. lossouarn@lecnam.net

†lucie.rouleau@lecnam. net

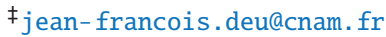



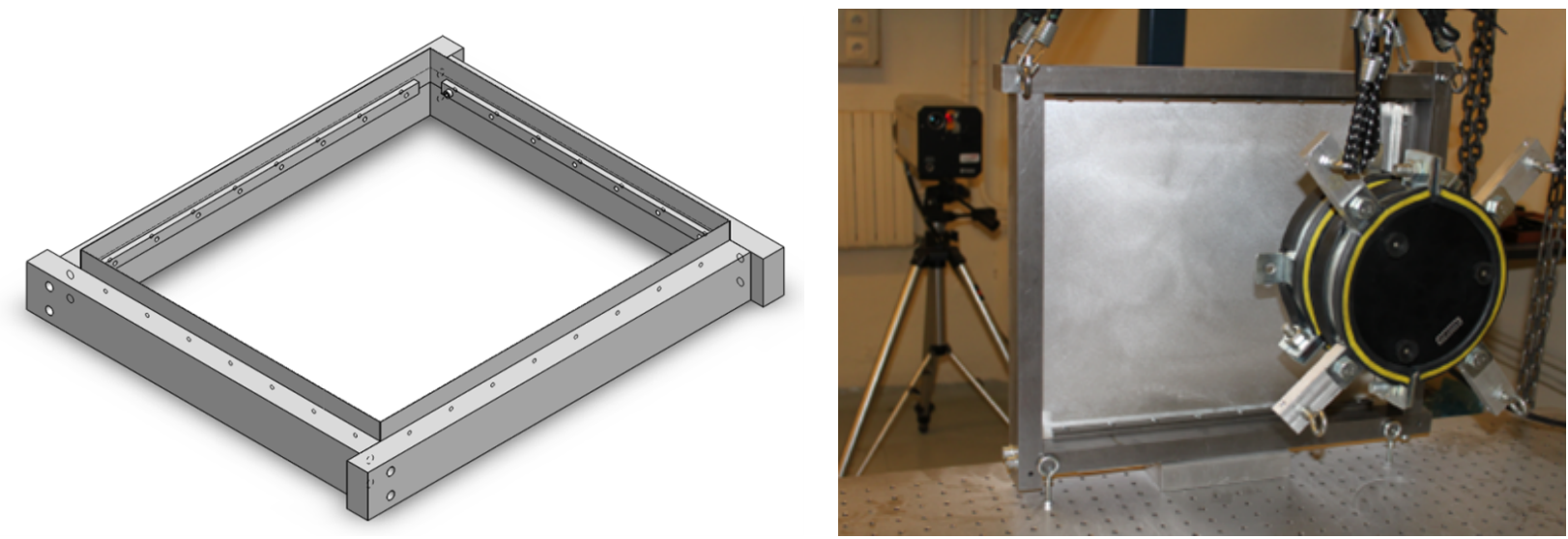

Fig. 1: Frame for the simply-supported boundary conditions and experimental setup.

constraints. While the present setup only offers a comparison for a single design of both plates, it still allows developing models for a more general purpose. This is the main objective of Section 3 that describes the models used for the following parametric studies. Both piezoelectric and viscoelastic models are first validated from the experiments and then used to provide several optimal configurations that differ from the initial setup. Subsequently, in Section 4, the two damping treatments can be compared on a more objective basis by considering equal-mass and equal-thickness configurations. Finally, with regard to this work, the advantages and limitations of viscoelastic or piezoelectric treatments are discussed.

\section{Benchmark for comparison of damping performance}

This section describes the geometry of the panels equipped with viscoelastic or piezoelectric patches and the experimental setup used to measure their structural dynamic response. Experimental results are reported and compared.

\subsection{Reference bare panel}

The reference panel is a simply-supported rectangular plate of lengths $l_{x}=420 \mathrm{~mm}, l_{y}=360 \mathrm{~mm}$ and thickness $h_{s}=3 \mathrm{~mm}$. It is made of 2017 aluminum alloy whose material properties are set to a Young's modulus $Y_{s}=69 \mathrm{GPa}$, a Poisson's ratio $v_{s}=0.346$ and a density $\rho_{s}=2800 \mathrm{~kg} / \mathrm{m}^{3}$. The simply-supported plate setup has been fully described by Robin et al. in $[18,19]$. As seen in Fig. $1,0.5 \mathrm{~mm}$ thick aluminum strips make the connection between the steel frame and the four edges of the plate on which they are glued. The strips have sufficient longitudinal stiffness to prevent transverse displacement while their low flexural stiffness approximates free rotational degrees of freedom.

In order to validate the simply-supported boundary conditions, vibration analysis of the aluminum plate mounted on the frame was carried out using a shaker and a scanning laser vibrometer (see Fig. 1). Table 1 shows experimental deflection shapes that highlight the combination of sinus-like shapes in both orthogonal directions such as classically observed with simply-supported plates. In Table 2, the experimental eigenfrequencies of the first ten undamped modes are compared to the theoretical eigenfrequencies obtained from

$$
f_{(m, n)}=h_{s}\left(\frac{m^{2}}{l_{x}^{2}}+\frac{n^{2}}{l_{y}^{2}}\right) \frac{\pi}{4} \sqrt{\frac{Y_{s}}{3 \rho_{s}\left(1-v_{s}^{2}\right)}} .
$$

A good correlation is observed between the experimental and theoretical results, which validates the assembly used to approximate the boundary conditions of a simply-supported plate. The largest error, of the order of $6 \%$ for the first mode, is explained by the presence of the strips on which the edges of the plate are glued. These strips are obviously not infinitely compliant in bending. Therefore, they only allow an approximation of the simply-supported boundary conditions, as explained by Robin et al. [18] who found a similar frequency error with a different plate setup. An experimental modal analysis also shows a parasitic mode around $685 \mathrm{~Hz}$. This corresponds to a bending mode of the frame that is not infinitely rigid. 


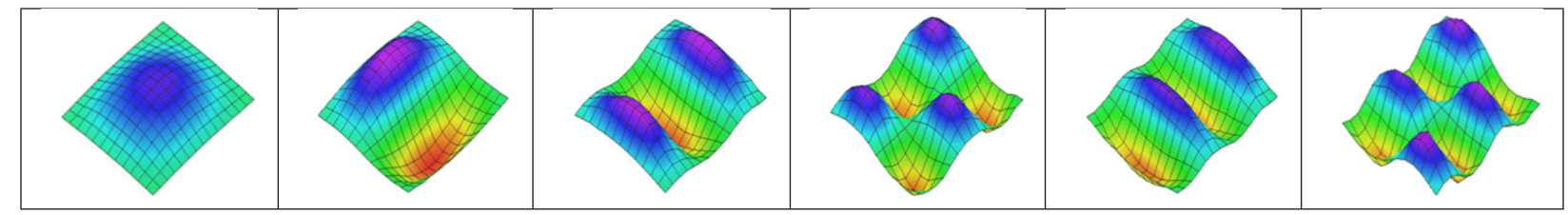

Table 1: Experimental operational deflection shapes of the simply-supported plate: $(1,1),(1,2),(3,1),(3,2),(4,1)$ and $(4,2)$ shapes, respectively from the left to the right.

\begin{tabular}{cccc}
\hline Mode & Experimental frequency $(\mathbf{H z})$ & Theoretical frequency $(\mathbf{H z})$ & Error $(\%)$ \\
\hline$(1,1)$ & 102.0 & 96.3 & 5.92 \\
$(2,1)$ & 224.2 & 218.7 & 2.51 \\
$(1,2)$ & 271.7 & 262.9 & 3.35 \\
$(2,2)$ & 392.8 & 385.3 & 1.95 \\
$(3,1)$ & 430.2 & 422.8 & 1.75 \\
$(1,3)$ & 547.9 & 540.6 & 1.35 \\
$(3,2)$ & 595.2 & 589.4 & 0.98 \\
$(2,3)$ & 668.8 & 663.0 & 0.87 \\
$(4,1)$ & 717.7 & 708.4 & 1.31 \\
$(3,3)$ & 871.5 & 867.1 & 0.51 \\
\hline \hline
\end{tabular}

Table 2: Comparison of the first ten experimental and theoretical eigenfrequencies for the bare panel.
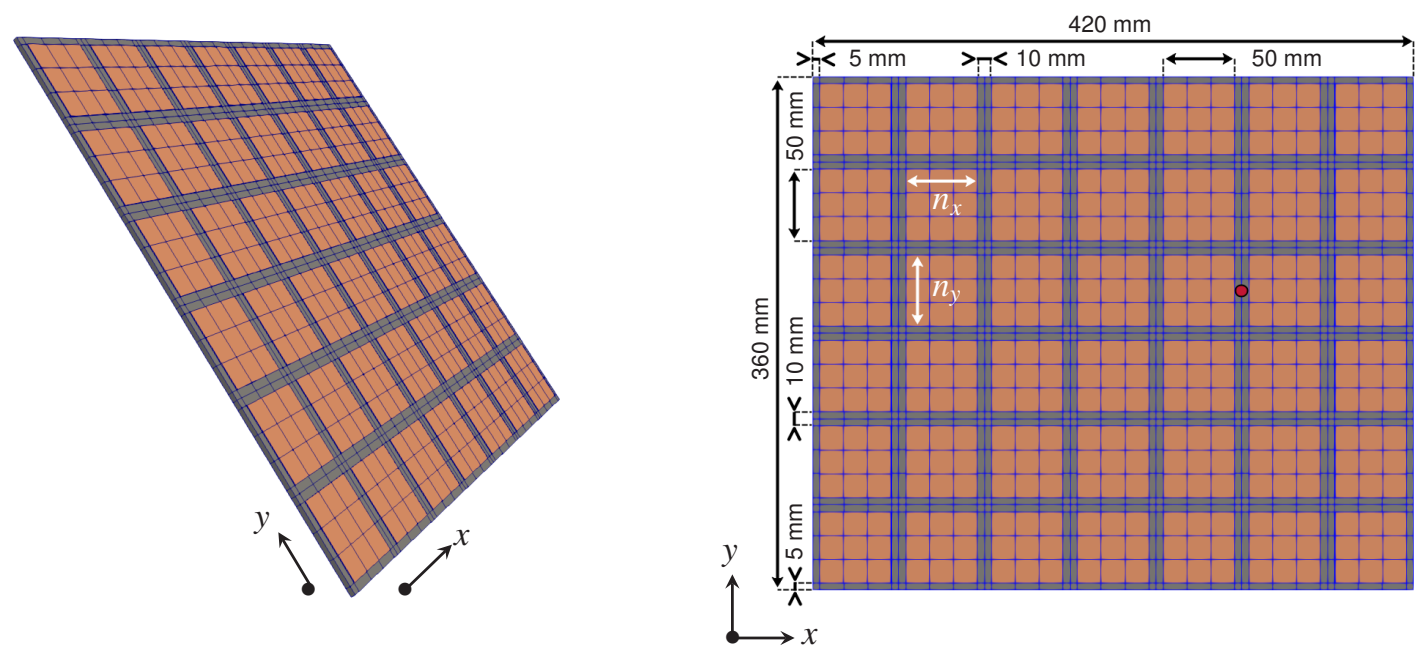

Fig. 2: Dimensions and meshing of the mechanical structure with 42 patches, and $\bullet(x, y)$ location of both the force and the acceleration measurements.

Once the simply-supported plate setup is validated, it can serve as a benchmark for comparison of damping treatments. The geometry and positioning of the damping elements added onto the surface of the bare panel has been driven by considerations related to the piezoelectric treatment. First, it was chosen to consider a broad frequency range up to $1 \mathrm{kHz}$, with more than ten different modes to be controlled simultaneously. As it can be predicted from the operational deflection shapes in Table 1, each mode would have its own optimal positioning of piezoelectric elements. However, considering a large number of bi-directional sinus-like modes with no priority given to any of them leads to a pragmatic solution consisting of covering the whole plate with piezoelectric patches. Then comes the problem of charge cancellation that requires dividing the piezoelectric layer into patches of small dimensions compared to the smallest wavelength to be controlled [20]. Even if an increased number of segments would maximize coupling for the highest modes, for practical reasons, the number of piezoelectric elements is here limited to 42 units, which allows the use of square patches as shown in Fig. 2. 
Journal of Structural Dynamics, 1, (pp. 30-48) 2021

Comparison of passive damping treatments based on constrained viscoelastic layers and multi-resonant piezoelectric networks
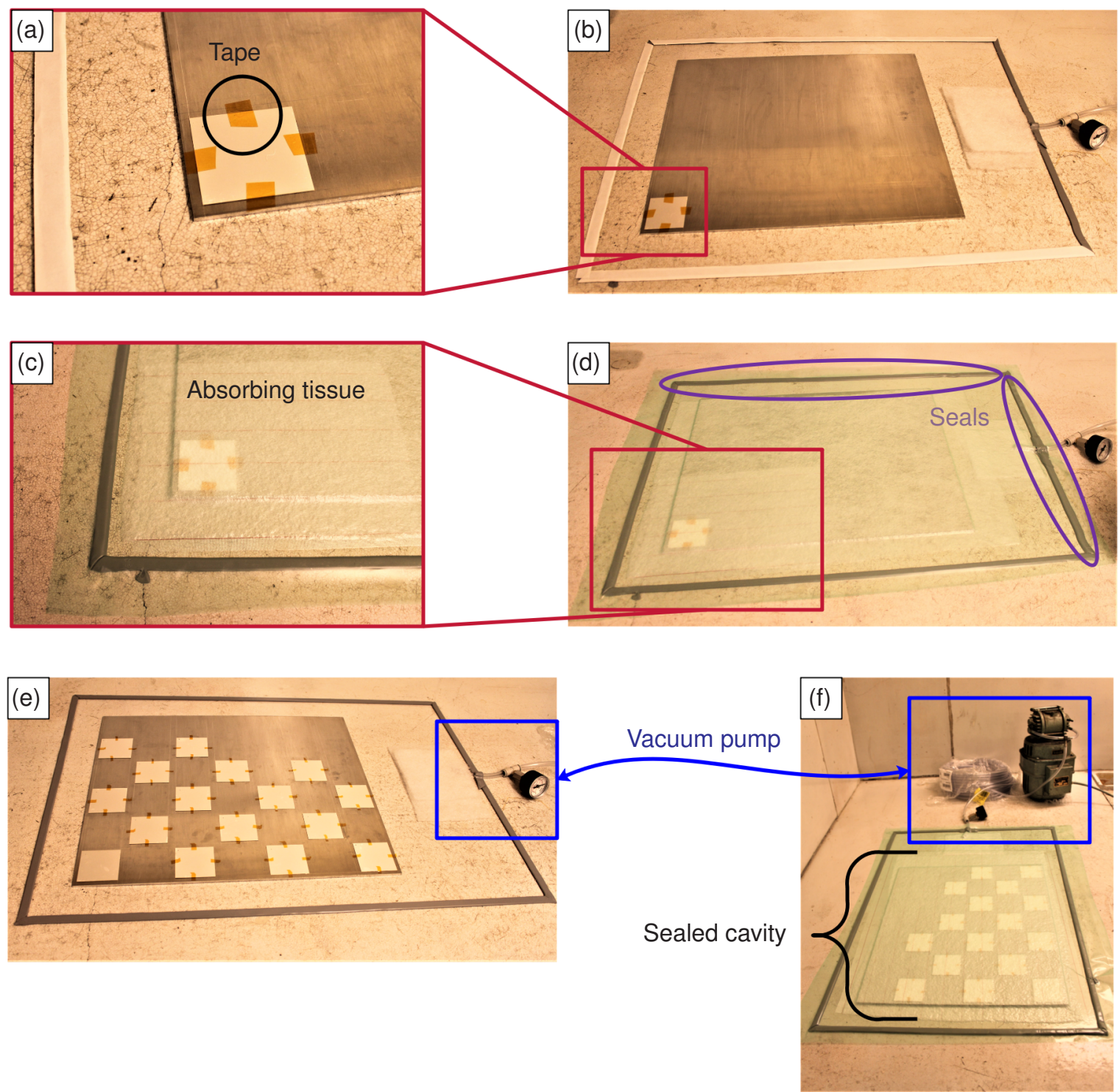

Fig. 3: Bonding of the piezoelectric patches on the plate: (a), (b) patch fixation using tape; (c) absorbing tissue between the plastic layer and the structure; (d) sealed cavity; (e) preparation for the bonding of several patches; (f) connection of the vacuum pump to the cavity.

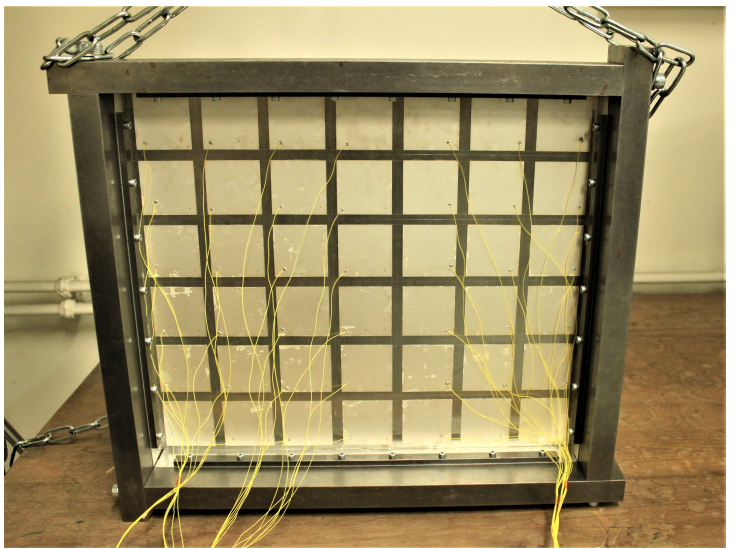

(a)

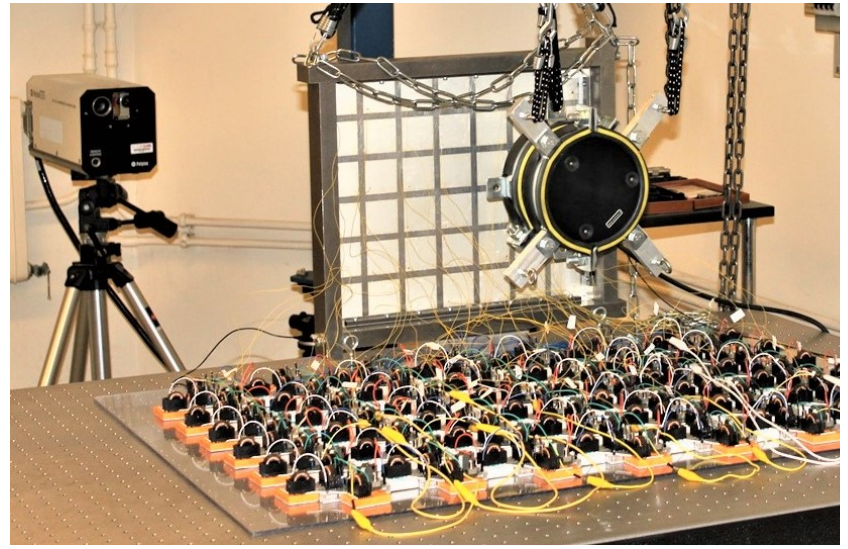

(b)

Fig. 4: (a) Rectangular panel covered by 42 square piezoelectric patches and (b) experimental setup including the analogous electrical network made of 42 identical unit cells. 


\subsection{Panel with piezoelectric patches}

Piezoelectric patches are bonded on one side of the plate as pictured in Fig. 3 showing the bonding process. A sealed cavity is formed around the structure with a thin layer of plastic material. A vacuum pump is then used to apply a load on the upper surfaces of the patches. The resulting layers of epoxy adhesive are sufficiently thin to be neglected during simulations. Moreover, it is experimentally observed that there is a short circuit between the aluminum panel and the lower electrodes of the piezoelectric patches. Since the epoxy material is non-conductive, this means that the proposed bonding process enforces a direct contact between some areas of the electrodes and the base panel.

The resulting panel covered with 42 square piezoelectric patches is pictured in Fig. 4. The sides and thicknesses of the piezoelectric transducers are respectively $l_{p}=50 \mathrm{~mm}$ and $h_{p}=0.5 \mathrm{~mm}$. They are made of PIC 153 piezoelectric material (PI Ceramic) which leads to a total of $400 \mathrm{~g}$, thus a $31 \%$ added mass compared to the $1270 \mathrm{~g}$ bare panel. Although not representative of an industrial application, this prototype piezoelectric plate gives access to model validation and further numerical extrapolation to cases with lower added mass.

The electrical network that interconnects the piezoelectric patches is fully described in [13]. It corresponds to an electrical analogue of the simply-supported plate. The piezoelectric patches whose blocked capacitance is around $C_{p}=140 \mathrm{nF}$ once glued are connected to 42 identical electrical unit cells made of passive components, including 42 inductors and 139 transformers. Their values and interconnections are chosen so as to create, in the electrical domain, the analogue of the plate to be controlled [21]. This generates a multi-resonant network whose natural frequencies are close to those of the mechanical plate in order to obtain the equivalent of a multimodal tuned mass damper.

As can be seen in Fig. 4b, the size of the electrical network is not negligible in the proposed experimental setup. However, it should be noted that this setup served as a proof of concept in terms of damping performance and was not intended for a demonstration of an integrated system. The mass of the electrical components, which can be set away from the plate thanks to potentially long wires, is thus not taken into account in this work. A choice of magnetic components for a specific application first requires to define the maximum vibration amplitudes to be controlled and the practical constraints for manufacturing of these components. On the other hand, the number of components can be greatly reduced if the control strategy focuses on a smaller number of modes, the limit case being the damping of a single mode by a resonant shunt which requires a single inductor.

\subsection{Panel with viscoelastic patches}

To test both passive damping treatments in the same configuration, 42 viscoelastic patches are also periodically distributed on a panel, as illustrated in Fig. 5 . Each $50 \mathrm{~mm} \times 50 \mathrm{~mm}$ patch is composed of a $120 \mu \mathrm{m}$ viscoelastic layer (Smacwrap $\AA$ ) and a $1.04 \mathrm{~mm}$ composite laminate which acts as a constraining layer. The properties of both materials are provided in Table 3. With these materials and geometry, the total mass of the 42 viscoelastic patches is $182 \mathrm{~g}$, resulting in a $14 \%$ added mass.

The patches were glued onto the plate using epoxy adhesive and vacuum bonding. The bonding process is realized in two steps to avoid bonding bridges between the constraining layer and the base panel. The measured thickness of each adhesive layer is comprised between $50 \mu \mathrm{m}$ and $100 \mu \mathrm{m}$ and will be neglected in numerical simulations.

\subsection{Experimental comparison of both damping treatments}

The steel frame is suspended with elastic straps and a shaker applies a pseudo-random point load to the plate. The mobility of the structure is obtained from an impedance head that measures the force and the acceleration at the excitation point. The location of both the force and the acceleration measurements is drawn in Fig. 2.

Both damping treatments are tested under the same experimental conditions: the panel with viscoelastic patches and the panel with piezoelectric patches connected to a multi-resonant network. The measured frequency response functions are plotted in Fig. 6. Both damping treatments induce a drastic reduction of the dynamic response over the

\begin{tabular}{cccc}
\hline Material & Young's modulus $(\mathrm{Pa})$ & Poisson's ratio & Density $\mathbf{( k g / \mathbf { m } ^ { 3 } )}$ \\
\hline Viscoelastic (static) & $2.9810^{6}$ & 0.49 & 867 \\
Laminate composite & $5.9410^{10}$ & 0.273 & 1580 \\
\hline
\end{tabular}

Table 3: Material properties considered in the finite element model of the sandwich panel. 


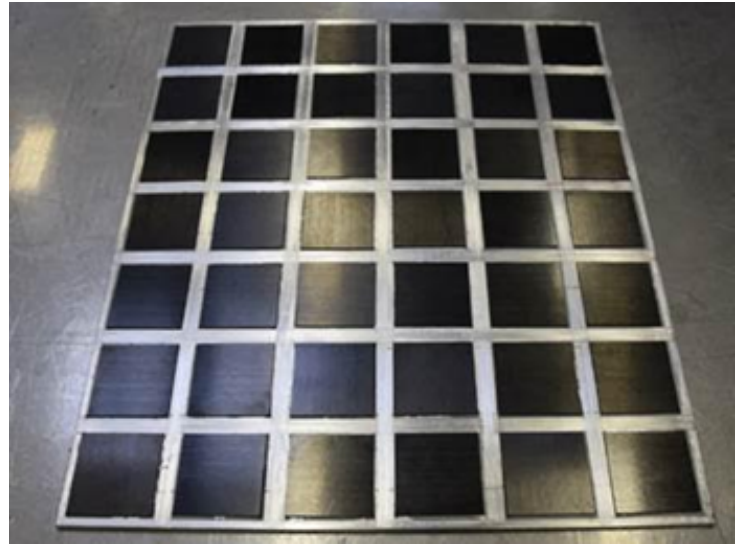

(a)

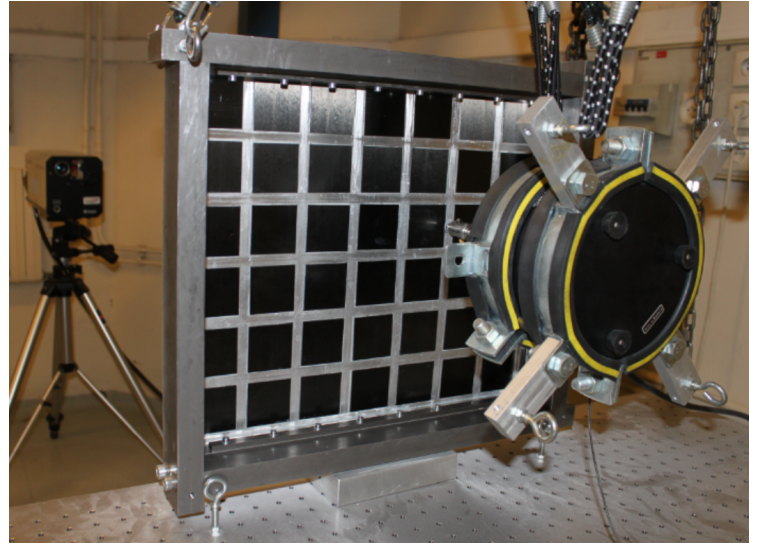

(b)

Fig. 5: (a) Panel covered with 42 constrained viscoelastic patches and (b) experimental setup for the viscoelastic case once the simply-supported panel is attached to the frame.

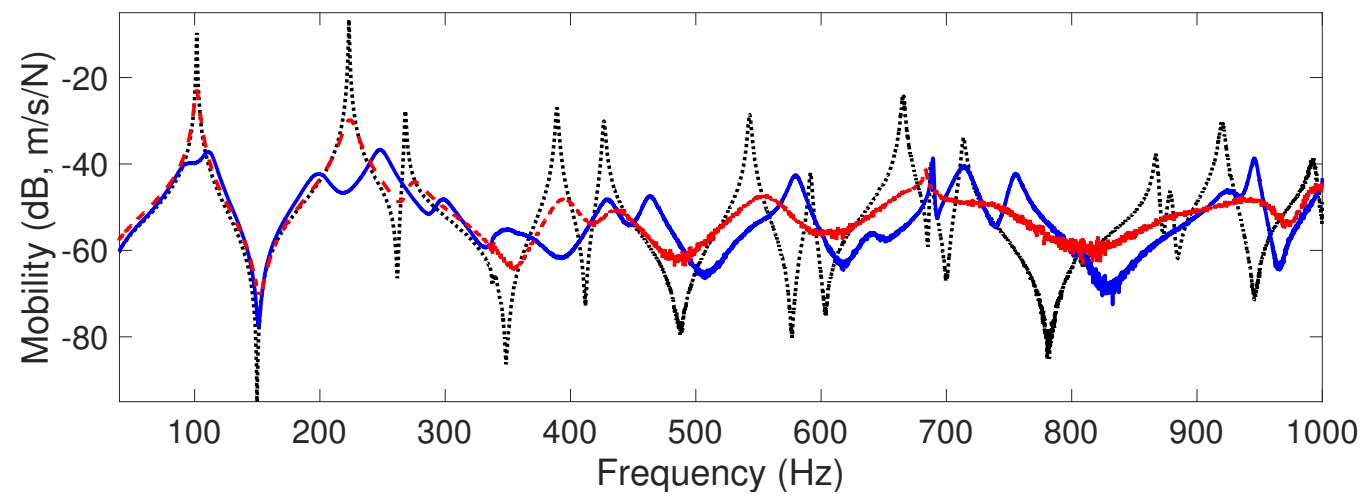

Fig. 6: Frequency response functions measured on the piezoelectric panel with short-circuited patches $(\cdots)$, the piezoelectric panel connected to the multi-resonant network $(-)$ and the panel with viscoelastic patches $(--)$.

whole frequency range. Multi-resonant piezoelectric damping is more efficient than viscoelastic damping for the first vibration modes. However, they offer similar damping performances above the fourth plate mode which is around $400 \mathrm{~Hz}$ with the present setup.

This experimental comparison is a necessary step in the evaluation of both damping techniques but it unfortunately represents a single design choice. Moreover, due to practical considerations, the plates equipped with piezoelectric and viscoelastic damping treatments were not designed according to equal-mass or equal-thickness configurations. In order to compare the two passive treatments in a more objective manner, numerical models of the damped panels need to be implemented and experimentally validated.

\section{Numerical models for parametric analysis}

This section describes the numerical models used to predict the dynamic response of a panel with piezoelectric or viscoelastic patches. Both models are validated by comparison with the experimental results presented in the previous section. The models are then used to evaluate the influence of different parameters such as the thickness of the layers or the tuning of the electrical network.

\subsection{Modelling of the panel with piezoelectric patches}

\subsubsection{Finite element model}

The main assumptions to derive the finite element formulation are: 


\begin{tabular}{|c|c|c|c|c|c|c|c|c|c|c|c|}
\hline \multicolumn{4}{|c|}{$\begin{array}{c}\text { Elastic } \\
\text { coefficients } \\
\left(10^{-12} \mathrm{~N} / \mathrm{m}^{2}\right)\end{array}$} & \multicolumn{2}{|c|}{$\begin{array}{l}\text { Poisson's } \\
\text { ratios } \\
(-)\end{array}$} & \multicolumn{2}{|c|}{$\begin{array}{l}\text { Piezoelectric } \\
\text { coefficients } \\
\left(10^{-12} \mathrm{C} / \mathrm{N}\right)\end{array}$} & \multicolumn{2}{|c|}{$\begin{array}{c}\text { Relative } \\
\text { permittivities } \\
(-)\end{array}$} & \multicolumn{2}{|c|}{$\begin{array}{l}\text { Density } \\
\left(\mathrm{kg} / \mathrm{m}^{3}\right)\end{array}$} \\
\hline$s_{11}^{E}$ & 16.83 & $s_{33}^{E}$ & $1.15 s_{11}^{E}$ & $v_{12}$ & 0.34 & $d_{31}$ & -260 & $\epsilon_{33}^{\sigma} / \epsilon_{0}$ & 4200 & $\rho_{p}$ & 7600 \\
\hline$s_{12}^{E}$ & $-v_{12} s_{11}^{E}$ & $s_{44}^{E}$ & $1.15 s_{66}^{E}$ & $v_{13}$ & $1.25 v_{12}$ & $d_{33}$ & $-2 d_{31}$ & $\epsilon_{33}^{\varepsilon} / \epsilon_{0}$ & 2575 & & \\
\hline$s_{13}^{E}$ & $-v_{13} s_{11}^{E}$ & $s_{66}^{E}$ & $2\left(s_{11}^{E}-s_{12}^{E}\right)$ & & & & & & & & \\
\hline
\end{tabular}

Table 4: PIC 153 PZT material characteristics used for the finite element modelling, where $\epsilon_{0}=8.854 \times 10^{-12} \mathrm{~F} / \mathrm{m}$ is the vacuum permittivity.

- The infinitesimal strain theory applies.

- The host structure is modelled as an isotropic homogeneous linear and undamped elastic medium.

- The thickness of the adhesive layers between the structure and the piezoelectric transducers is neglected.

- The piezoelectric transducers are thin and polarized in the direction orthogonal to the electrodes.

- The electrode thickness is neglected, and those on the side of the panel are connected to the ground.

- There are no free electric charges on the sides of the piezoelectric patches.

- The electric characteristic time in the piezoelectric domain is much smaller than the mechanical one, hence the electric field $\mathbf{E}$ is described in terms of a scalar potential $V$.

Under these assumptions, Thomas et al. [22] showed that a structure covered by thin piezoelectric patches can be modelled with only one electrical degree of freedom per piezoelectric patch, as equipotentiality is ensured over the electrodes. Therefore, the voltage on the upper electrode of each piezoelectric patch is a global variable. If we note $\mathbf{U}$ the vector containing the nodal values of the displacement field and $\mathbf{V}=\left(V^{(1)}, \ldots, V^{(p)}\right)$ the vector containing the electric potential values on the upper electrodes of the piezoelectric patches, the electromechanical finite element model can be written as

$$
\left(\begin{array}{cc}
\mathbf{M}_{\mathbf{m}} & \mathbf{0} \\
\mathbf{0} & \mathbf{0}
\end{array}\right)\left(\begin{array}{c}
\ddot{\mathbf{U}} \\
\ddot{\mathbf{Q}}
\end{array}\right)+\left(\begin{array}{cc}
\mathbf{K}_{\mathbf{m}}+\mathbf{K}_{\mathbf{c}} \mathbf{K}_{\mathbf{e}} \mathbf{K}_{\mathbf{c}}^{\top} & \mathbf{K}_{\mathbf{c}} \mathbf{K}_{\mathbf{e}} \\
\left(\mathbf{K}_{\mathbf{c}} \mathbf{K}_{\mathbf{e}}\right)^{\top} & \mathbf{K}_{\mathbf{e}}
\end{array}\right)\left(\begin{array}{l}
\mathbf{U} \\
\mathbf{Q}
\end{array}\right)=\left(\begin{array}{l}
\mathbf{F} \\
\mathbf{V}
\end{array}\right) .
$$

With these notations, $\mathbf{F}$ contains the external mechanical forces applied to the structure, while in $\mathbf{Q}=\left(Q^{(1)}, \ldots, Q^{(p)}\right)$ are the electrical charges on the transducers electrodes. $\mathbf{M}_{\mathbf{m}}$ and $\mathbf{K}_{\mathbf{m}}$ are the mechanical mass and stiffness matrices, respectively and $\mathbf{K}_{\mathbf{c}}$ is the coupling matrix. $\mathbf{K}_{\mathbf{e}}$ is a diagonal matrix in which the $j$-th term is the inverse of the blocked piezoelectric capacitance.

The main aluminum plate is modelled with 20-node hexahedral elements. Both the plate and the piezoelectric patches are meshed with one element in depth. In the other directions, the piezoelectric patches as well as the plate beneath them are meshed with $n_{x} \times n_{y}$ elements. Considering $n_{x}=n_{y}=3$ leads to converged values for natural frequencies of the undamped structure up to $1 \mathrm{kHz}$. The maximum remaining difference between natural frequencies estimated with $n_{x}=n_{y}=3$ and $n_{x}=n_{y}=5$ is $0.4 \%$. Accordingly, Fig. 2 depicts the mesh used to obtain all the following numerical results.

The piezoelectric patches are made of the PIC 153 PZT material. As few material characteristics are available in the manufacturer's data, some are either extrapolated from datasheets of other PZT materials, or numerically optimized [13]. All the numerical values finally used for the modelling are summed up in Table 4.

\subsubsection{Validation of the piezoelectric numerical model}

For any $n$-th mode of the piezoelectric structure, a modal coupling factor $\left(k_{c}\right)_{n}$ can be defined as

$$
\left(k_{c}\right)_{n}=\sqrt{\left(\frac{\left(f_{\mathrm{OC}}\right)_{n}}{\left(f_{\mathrm{SC}}\right)_{n}}\right)^{2}-1} .
$$




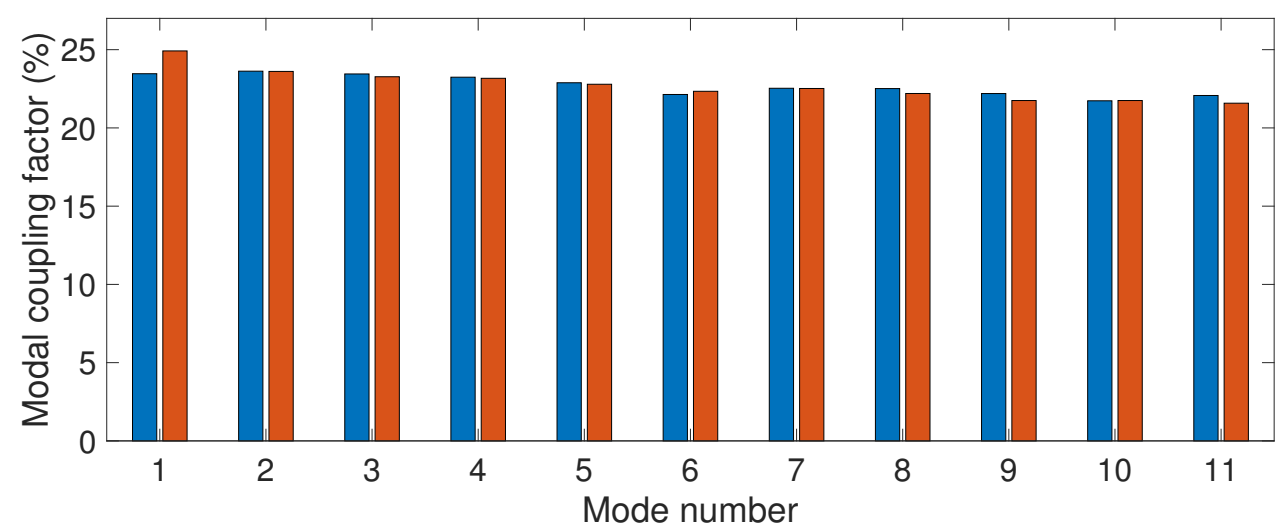

Fig. 7: Comparison of the modal coupling factors for the first eleven plate modes: experimental (left bars) and numerical (right bars).

In this expression, $\left(f_{\mathrm{SC}}\right)_{n}$ is the $n$-th natural frequency of the plate when the piezoelectric patches are short-circuited, while $\left(f_{\mathrm{OC}}\right)_{n}$ is obtained when each upper electrode of the piezoelectric patches is left disconnected. These frequencies are estimated by using the finite element formulations of Eq. (2) with $\mathbf{V}=\mathbf{0}$ or $\mathbf{Q}=\mathbf{0}$, so the mechanical eigenvalue problem is solved with stiffness matrices $\mathbf{K}_{\mathbf{m}}$ or $\mathbf{K}_{\mathbf{m}}+\mathbf{K}_{\mathbf{c}} \mathbf{K}_{\mathbf{e}} \mathbf{K}_{\mathbf{c}}{ }^{\top}$, respectivelly. As a result, the simulated coupling factors are shown in Fig. 7. They are compared to the experimental ones that were obtained from a Rational Fraction Polynomial method [23] for the identification of the open- and short-circuit natural frequencies. The gap between the simulated and measured coupling factors is less than $5 \%$. Besides, this maximum overestimation is for the first coupling factor and could be partly explained by the non-ideal experimental boundary conditions, especially for the first mode.

Once the model is validated in terms of resulting coupling factors, it can be used to predict damping performance once the piezoelectric plate is connected to electrical components. As fully described in [13], interconnecting the piezoelectric elements with an electrical network adds new matrix relations linking the piezoelectric charge vector $\mathbf{Q}$ and the piezoelectric voltage vector $\mathbf{V}$ to a network charge vector $\mathbf{Q}_{\mathbf{N}}$ and a forcing voltage vector $\mathbf{V}_{\mathbf{N}}$ related to the degrees of freedom of the considered electrical network. The electromechanical finite element model is finally described by the system

$$
\left(\begin{array}{cc}
\mathbf{M}_{\mathbf{m}} & \mathbf{0} \\
\mathbf{0} & \mathbf{M}_{\mathbf{N}}
\end{array}\right)\left(\begin{array}{c}
\ddot{\mathbf{U}} \\
\ddot{\mathbf{Q}}_{\mathrm{N}}
\end{array}\right)+\left(\begin{array}{cc}
\mathbf{0} & \mathbf{0} \\
\mathbf{0} & \mathbf{C}_{\mathbf{N}}
\end{array}\right)\left(\begin{array}{c}
\dot{\mathbf{U}} \\
\dot{\mathbf{Q}}_{\mathrm{N}}
\end{array}\right)+\left(\begin{array}{cc}
\mathbf{K}_{\mathrm{m}}+\mathbf{K}_{\mathbf{c}} \mathbf{K}_{\mathbf{e}} \mathbf{K}_{\mathbf{c}}^{\top} & \mathbf{K}_{\mathbf{c}} \mathbf{K}_{\mathbf{e}} \mathbf{P} \\
\left(\mathbf{K}_{\mathbf{c}} \mathbf{K}_{\mathbf{e}} \mathbf{P}\right)^{\top} & \mathbf{K}_{\mathbf{N}}
\end{array}\right)\left(\begin{array}{c}
\mathbf{U} \\
\mathbf{Q}_{\mathbf{N}}
\end{array}\right)=\left(\begin{array}{c}
\mathbf{F} \\
\mathbf{V}_{\mathbf{N}}
\end{array}\right)
$$

where $\mathbf{P}$ is a connectivity matrix that only depends on the topology of the electrical network and $\mathbf{M}_{\mathbf{N}}$ and $\mathbf{K}_{\mathbf{N}}$ can be seen as the electrical "inertia" and "stiffness" matrices. Since the objective of the present study is to provide vibration damping, resistive components are required and their influence can be modelled by a damping matrix $\mathbf{C}_{\mathbf{N}}$ that is added to the electrical degrees of freedom in Eq. (4), while neglecting the purely mechanical damping.

After projection on the first 100 modes of the electromechanical system, the numerical frequency response function is compared to the experimental one. The results are shown in Fig. 8 which validates the numerical model. The main differences are actually due to the slight imperfections of the panel setup (approximation of simply-supported boundary conditions and undesired frame modes) but not directly to the modelling of the electromechanical coupling.

\subsubsection{Optimization of the piezoelectric network}

The experimental frequency response function is not the result of a strict optimization process of the assembled electrical network. This network had been designed with a constraint on sufficiently low electrical damping in order to observe an underdamped behavior of the proposed tuned vibration absorber around the first mode. Even if the multi-resonant network was intended for broadband vibration mitigation, during the design phase no specific constraint had been fixed for broadband optimization. Resistance can yet be easily added to the network afterwards in order to minimize specific ranges of the frequency response function or to maximize damping ratios. In the following, we choose to focus on the maximization of the damping ratios.

The numerical model is used to extract the damping ratios of the electromechanical system for various tuning configurations of the electrical components. However, Eq. (4) cannot be directly used to compute undamped modes and a diagonal modal damping matrix because the transformers used in the electrical network induce non-proportional 

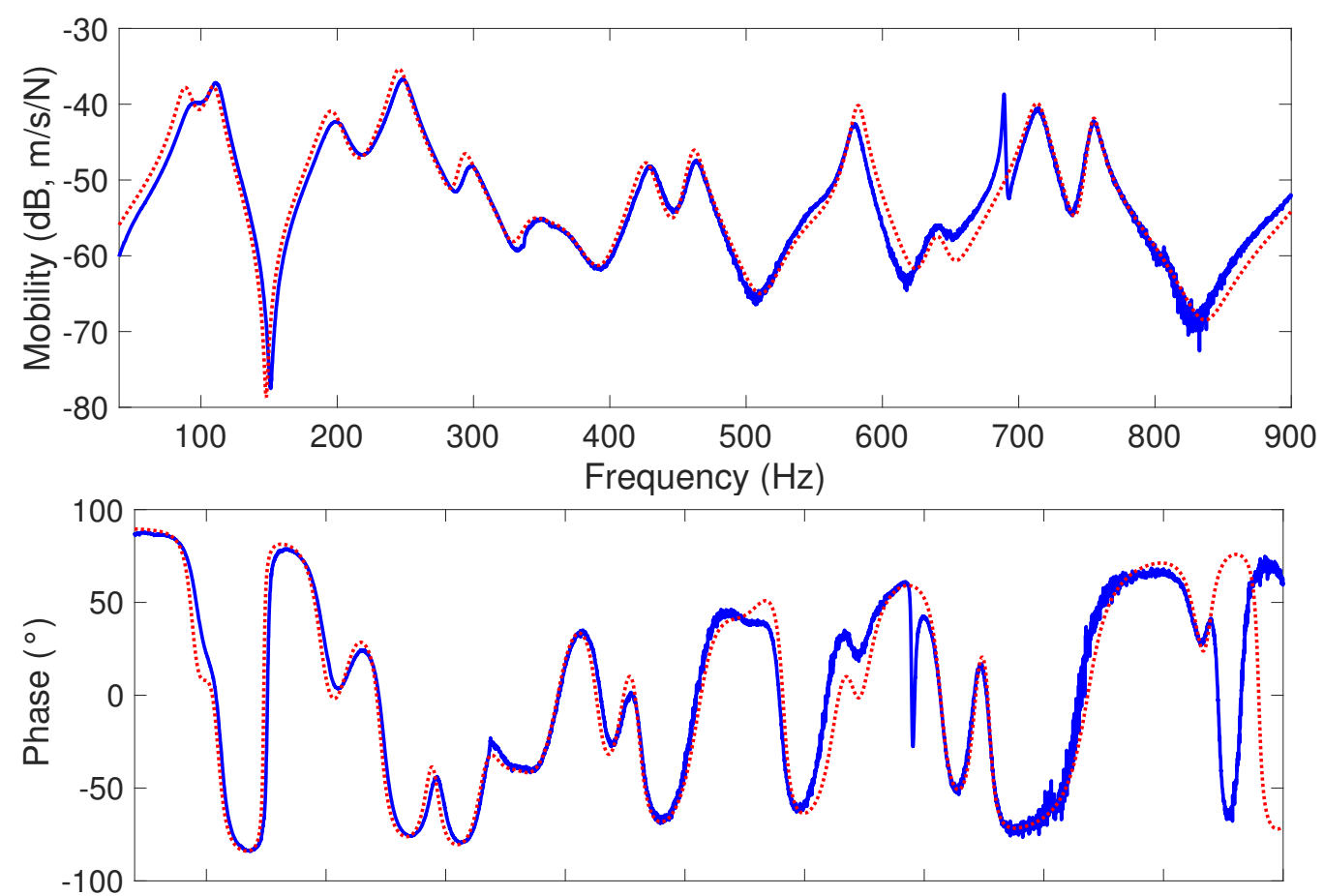

Fig. 8: Experimental (-) and numerical $(\cdots)$ mobilities for the panel with piezoelectric patches connected to a multi-resonant network.

damping. To solve this problem, the use of a state-space formulation is required [24, 25]. The application of the method to the considered electromechanical system leads to the following matrix formulation:

$$
\left(\begin{array}{cccc}
\mathbf{0} & \mathbf{0} & \mathbf{M}_{\mathbf{m}} & \mathbf{0} \\
\mathbf{0} & \mathbf{C}_{\mathbf{N}} & \mathbf{0} & \mathbf{M}_{\mathbf{N}} \\
\mathbf{M}_{\mathbf{m}} & \mathbf{0} & \mathbf{0} & \mathbf{0} \\
\mathbf{0} & \mathbf{M}_{\mathbf{N}} & \mathbf{0} & \mathbf{0}
\end{array}\right)\left(\begin{array}{c}
\dot{\mathbf{U}} \\
\dot{\mathbf{Q}}_{\mathrm{N}} \\
\ddot{\mathbf{U}} \\
\ddot{\mathbf{Q}}_{\mathrm{N}}
\end{array}\right)+\left(\begin{array}{cccc}
\mathbf{K}_{\mathbf{m}}+\mathbf{K}_{\mathbf{c}} \mathbf{K}_{\mathbf{e}} \mathbf{K}_{\mathbf{c}}^{\top} & \mathbf{K}_{\mathbf{c}} \mathbf{K}_{\mathbf{e}} \mathbf{P} & \mathbf{0} & \mathbf{0} \\
\left(\mathbf{K}_{\mathbf{c}} \mathbf{K}_{\mathbf{e}} \mathbf{P}\right)^{\top} & \mathbf{K}_{\mathbf{N}} & \mathbf{0} & \mathbf{0} \\
\mathbf{0} & \mathbf{0} & -\mathbf{M}_{\mathbf{m}} & \mathbf{0} \\
\mathbf{0} & \mathbf{0} & \mathbf{0} & -\mathbf{M}_{\mathbf{N}}
\end{array}\right)\left(\begin{array}{c}
\mathbf{U} \\
\mathbf{Q}_{\mathrm{N}} \\
\dot{\mathbf{U}} \\
\dot{\mathbf{Q}}_{\mathrm{N}}
\end{array}\right)=\left(\begin{array}{c}
\mathbf{0} \\
\mathbf{0} \\
\mathbf{0} \\
\mathbf{0}
\end{array}\right) .
$$

By computing the complex eigenvalues $\lambda_{i}$ that are defined as

$$
\lambda_{i}=-\xi_{i} \omega_{i} \pm \mathrm{j} \omega_{i} \sqrt{1-\xi_{i}^{2}},
$$

one can obtain the natural angular frequencies $\omega_{i}$ and damping ratios $\xi_{i}$ from

$$
\omega_{i}=\left|\lambda_{i}\right| \quad \text { and } \quad \xi_{i}=-\frac{\operatorname{Re}\left(\lambda_{i}\right)}{\left|\lambda_{i}\right|} .
$$

The eleven lowest damping ratios over a frequency band up to $1 \mathrm{kHz}$ are represented in Fig. 9. For the case modelling the experimental setup, the first damping ratios are around $5 \%$ but they drop to less than $1 \%$ for the highest modes. However, the network was designed for an approximate $\mathcal{H}^{\infty}$ optimum on the first mode but not a maximization of the damping ratios. This requires increased resistance in the network $[6,7,4]$ and selection of one specific mode for the optimization. Due to the relatively coarse mesh used to create the electrical analogue (42 unit cells), the proposed multi-resonant network is indeed not able to provide a perfect matching for all the modes over the considered frequency range. Although homogenized models can lead to the definition of optimal electrical parameters for broadband damping [9], the use of discrete electrical networks requires numerical optimization with precedence given to a specific mode in the considered frequency range. A first case targets the second mode of the undamped panel. The inductance in all the unit cells of the network is decreased from $244 \mathrm{mH}$ to $219 \mathrm{mH}$ and the transformer resistance is increased from $278 \Omega$ to $2350 \Omega$. This results in a strong increase of the damping ratio, especially for the first modes of the structure, as seen in Fig. 9. The effect of the network tuning also has a strong impact on the frequency response function represented in Fig. 10. Note that an increase of the damping ratio is not equivalent to a decrease of the vibration amplitude. This can be easily observed for the first mode, where two close modes 


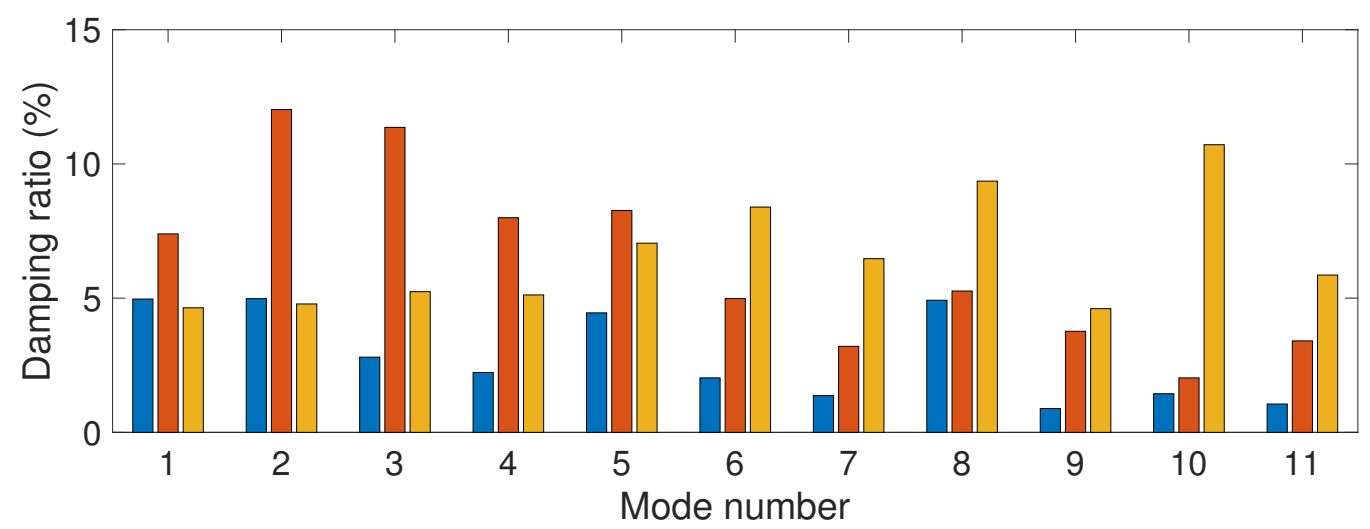

Fig. 9: Damping ratios extracted from the model of the reference experimental case (left bars), after maximization of the damping ratio on mode 2 (central bars) and on mode 10 (right bars).

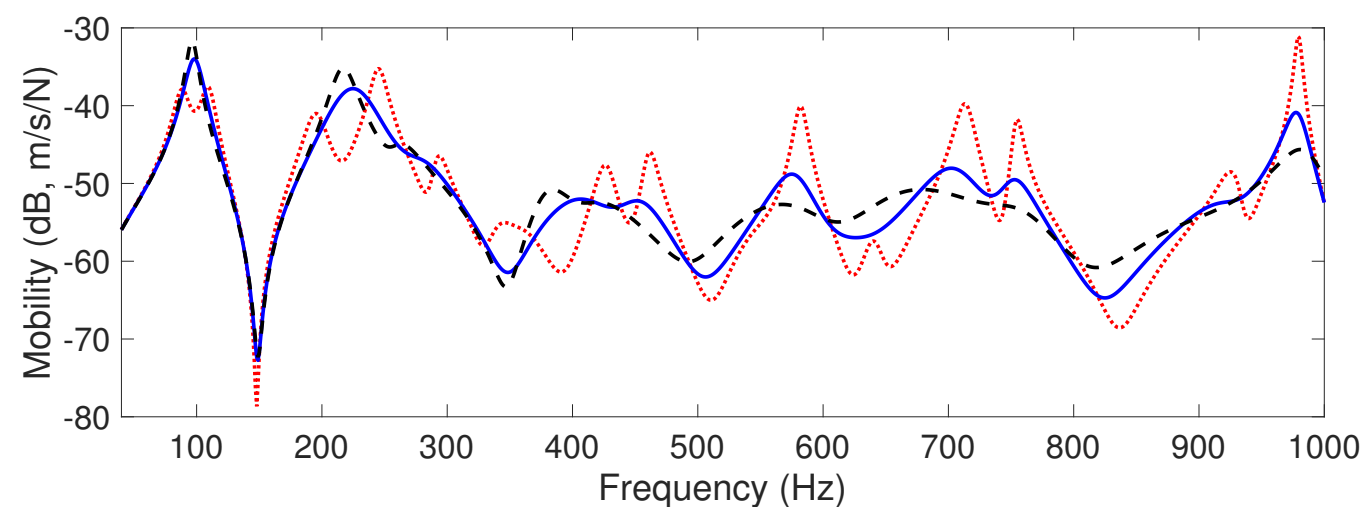

Fig. 10: Numerical frequency response functions for the reference experimental case $(\cdots)$, after maximization of the damping ratio on mode $2(-)$ and on mode $10(--)$.

merge into a double mode whose amplitude is the sum of two contributions. The vibration mitigation effect on the forced response is however significant for the highest modes even if the target mode was the second one because the increase of damping has a positive impact on the whole frequency range. If the highest modes are of prime interest, another mode can be used in the optimization of damping ratios. For example, with a $171 \mathrm{mH}$ inductance and a $2040 \Omega$ transformer resistance, one moves the maximal damping ratio from mode 2 to mode 10 , as seen in Fig. 9. This new optimization is at the expense of vibration mitigation performance over the first modes but it keeps flattening the frequency response function in the second half of the frequency range.

In the end, these results highlight a strong interest of piezoelectric network damping that is the possibility to tune the values of the electrical components depending on the considered objective function. The objective can either be minimization of a forced response over a predefined frequency range or maximization of specific damping ratios.

\subsubsection{Influence of the piezoelectric layer thickness}

As seen above, the tuning of the electrical network is of prime interest for maximizing the damping ratios but another fundamental parameter is the thickness of the piezoelectric layer. Actually, the amount of piezoelectric material has a direct impact on the modal coupling factors, which are related to the maximum attainable damping ratios. It can be shown from a one-degree-of-freedom approximation around the $n$-th resonance [7], that optimal resistive and resonant piezoelectric shunts lead to the following modal damping ratios $\left(\left(\xi_{\mathrm{R}}^{\mathrm{opt}}\right)_{n}\right.$ and $\left(\xi_{\mathrm{RL}}^{\mathrm{opt}}\right)_{n}$, respectively) for moderate modal coupling factors $\left(k_{c}<0.3\right)$ :

$$
\left(\xi_{\mathrm{R}}^{\mathrm{opt}}\right)_{n} \approx \frac{\left(k_{c}\right)_{n}^{2}}{4} \quad \text { and } \quad\left(\xi_{\mathrm{RL}}^{\mathrm{opt}}\right)_{n} \approx \frac{\left(k_{c}\right)_{n}}{2} .
$$

In the present case, these resistive and resonant shunts could be implemented with identical independent shunts connected to the 42 piezoelectric patches but an optimized interconnected network can provide similar damping. 


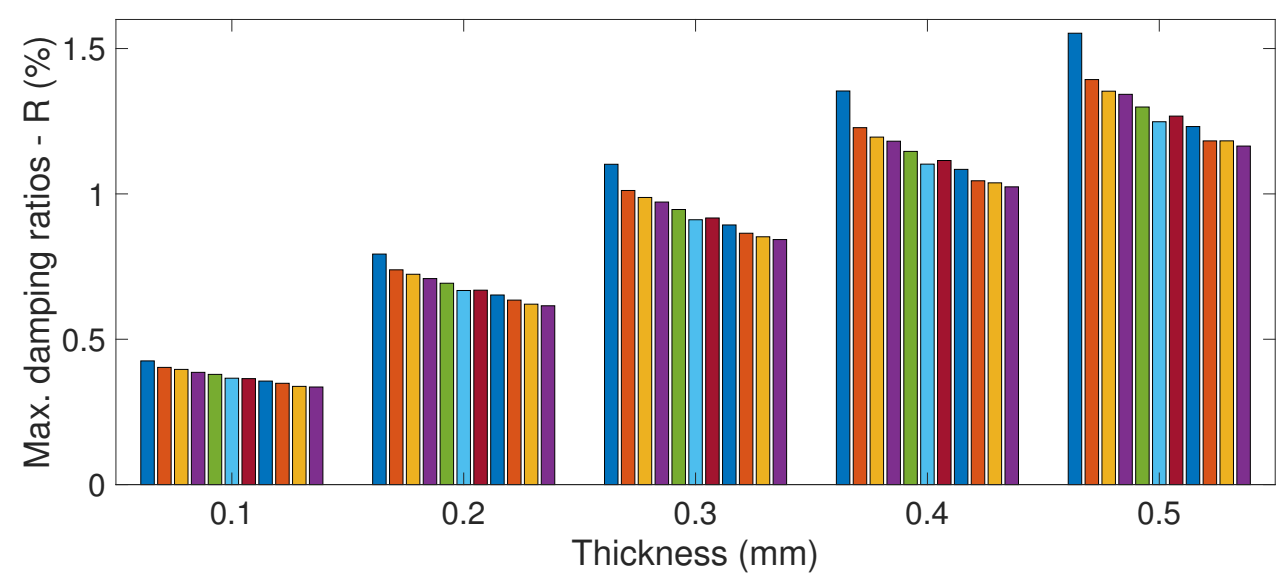

Fig. 11: Maximum added damping that would be achieved with an optimal resistive network $(R)$ for the first eleven modes with varying thickness of the piezoelectric patches.

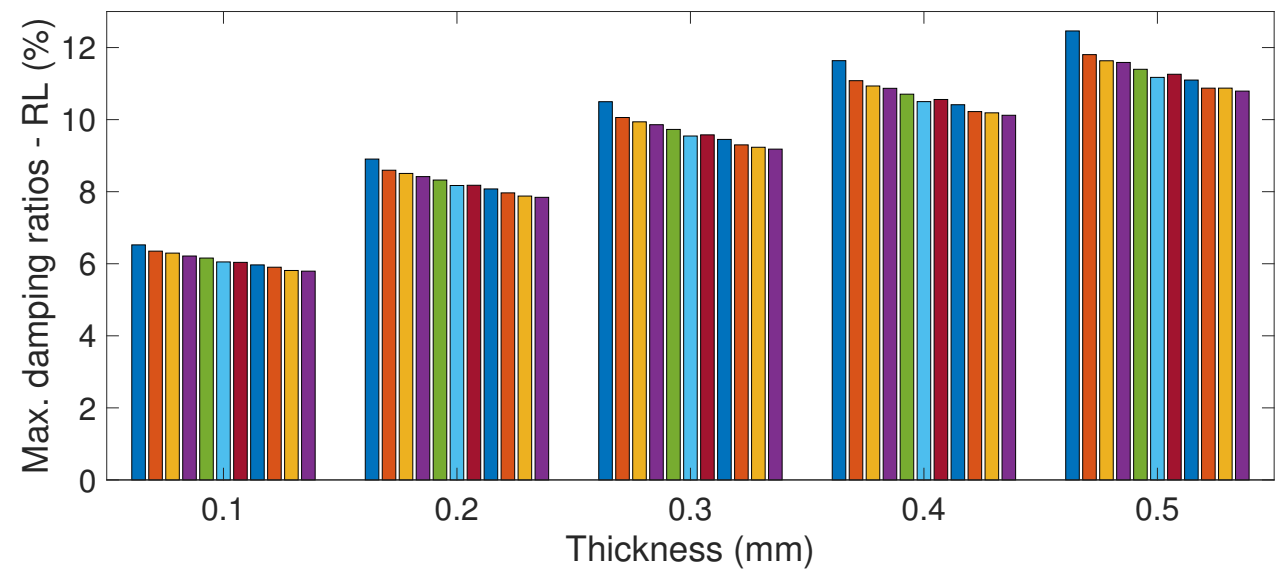

Fig. 12: Maximum added damping that would be achieved with an optimal resonant network $(R L)$ for the first eleven modes with varying thickness of the piezoelectric patches.

Note that the examples on resistive and resonant shunts are only considered in the present subsection in order to extract the maximum attainable damping from classical results related to single mode analysis (see Eq. (8)). This provides realistic targets for all the next simulations optimizing multi-resonant piezoelectric networks similar to the experimental one shown in Fig. 4b and fully detailed in [13]. The optimal damping ratios can be computed from the coupling factors in Eq. (3) by considering different values for the thickness of the piezoelectric layer, still modelled with one finite element in the depth direction. Figures (11) and (12) show the results for the first eleven modes of the proposed numerical model. One can remark that, for the case here considered, damping offered by a resonant network is about one order of magnitude larger than damping offered by a resistive network. This result justifies the focus on resonant networks in the present study. Secondly, $\xi_{\mathrm{R}}$ is approximately doubled and $\xi_{\mathrm{RL}}$ multiplied by a factor $\sqrt{2}$ when the thickness is doubled. Indeed, multiplying the modal fraction of strain energy by 2 leads to a $\sqrt{2}$ increase of the coupling factor [4]. This interpretation is only valid for sufficiently thin piezoelectric layers but it represents a useful approximation for most cases considering a limited quantity of piezoelectric material compared to the host structure.

A limit of the results in Figs. (11) and (12) is the fact that these maximum damping ratios cannot be obtained simultaneously with the previously described electrical network. Because the discrete electrical analogue cannot perfectly match all the mechanical resonances of the continuous structure, it is required to optimize damping on one specific mode and let the other ones divert from their optimal values. New resonant network layouts offering optimal tuning on a specified set of modes [10,12] are currently under investigation in order to reach the maximum attainable damping in Fig. 12 simultaneously for all the chosen modes. 


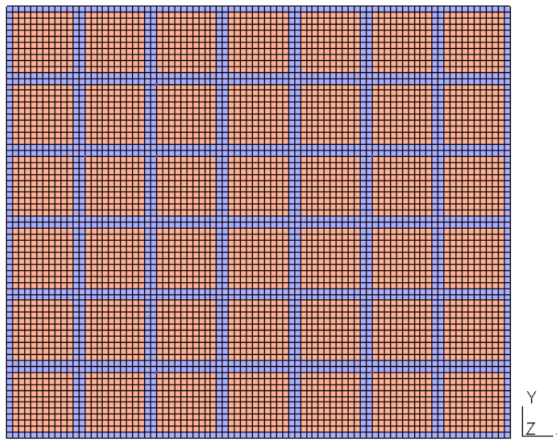

(a)

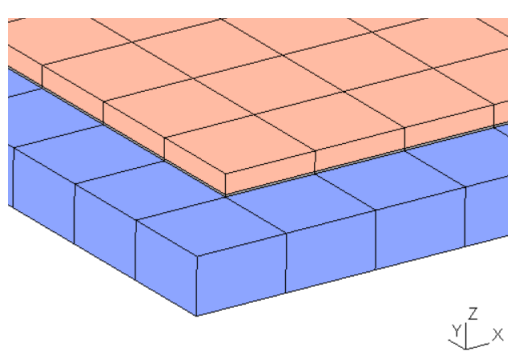

(b)

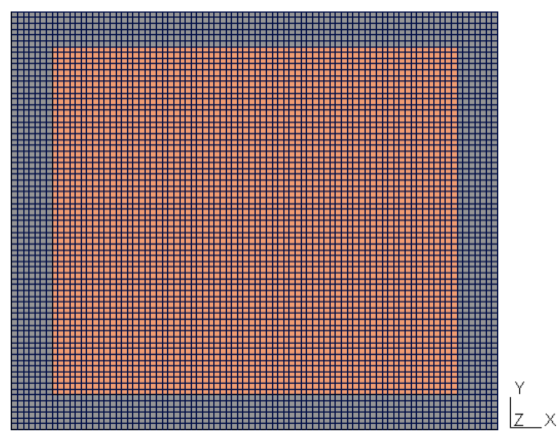

(c)

Fig. 13: Finite element mesh of the plate with viscoelastic patches: (a) with 42 patches and (c) with a single patch . (b) Close up of the finite element model.

\subsection{Modelling of the panel with viscoelastic patches}

\subsubsection{Finite element model}

A finite element model of the panel with viscoelastic patches is implemented. The main underlying assumptions are:

- The infinitesimal strain theory applies.

- The host structure, the viscoelastic layer and the constraining layer are modelled as isotropic homogeneous linear elastic materials (the stacking sequence of the composite laminate is such that the constraining layer is quasi-isotropic).

- The adhesive layers between the different layers are not modeled since they do not modify significantly the computed dynamic response.

- The damping of the host structure and the constraining layer is neglected compared to the inherent damping of the viscoelastic layer.

- The Poisson's ratio of the viscoelastic layer is assumed constant.

Under these assumptions, the finite element model can be written as:

$$
\left(\mathbf{K}_{f}+G^{*}(\omega) \mathbf{K}_{v}-\omega^{2} \mathbf{M}\right) \mathbf{U}=\mathbf{F},
$$

where $\mathbf{K}_{f}$ is the stiffness matrix associated to the base panel and the constraining layer, $\mathbf{K}_{v}$ is the stiffness matrix associated to the viscoelastic layer, $\mathbf{M}$ is the mass matrix of the whole structure, $\mathbf{U}$ is the vector of nodal displacements and $\mathbf{F}$ contains the external mechanical forces applied to the structure. $G^{*}(\omega)$ is the frequency-dependent shear modulus of the viscoelastic layer.

The whole structure is meshed with 20-node hexahedron elements, so that the shear behavior of the viscoelastic layer is properly represented and locking effects are avoided. Each layer is meshed with one finite element through the depth, and the size of the finite elements in the two other directions is kept constant in all the numerical simulations (see Fig. 13).

The material properties considered in the calculation are reported in Table 3, and the master curves of the viscoelastic layer, made of Smacwrap ${ }^{\circledR}$, are plotted in Fig. 14. The frequency dependent properties of the viscoelastic layer are taken into account in the finite element model through tabular data.

\subsubsection{Validation of the viscoelastic numerical model}

Figure 15 compares the frequency response function computed with the proposed finite element model to the response experimentally measured on the panel equipped with viscoelastic patches. An overall good agreement is observed between the numerical and experimental results, despite a small shift both in frequency and in amplitude. The highest error on the eigenfrequency, of $7.8 \%$, concerns the first vibration mode and can be related in part to the experimental frame [19], since a typical $5 \%$ error is observed on the first natural frequency with this kind of setup. 

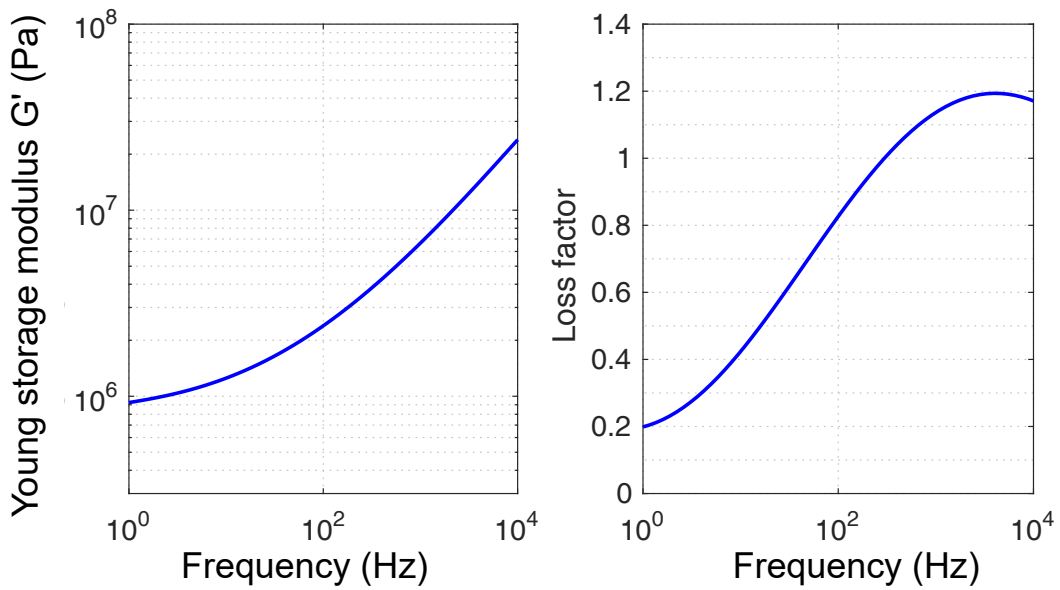

Fig. 14: Master curves of the viscoelastic material $(\mathrm{Smacwrap} \AA)$ at the reference temperature $T=20^{\circ} \mathrm{C}$
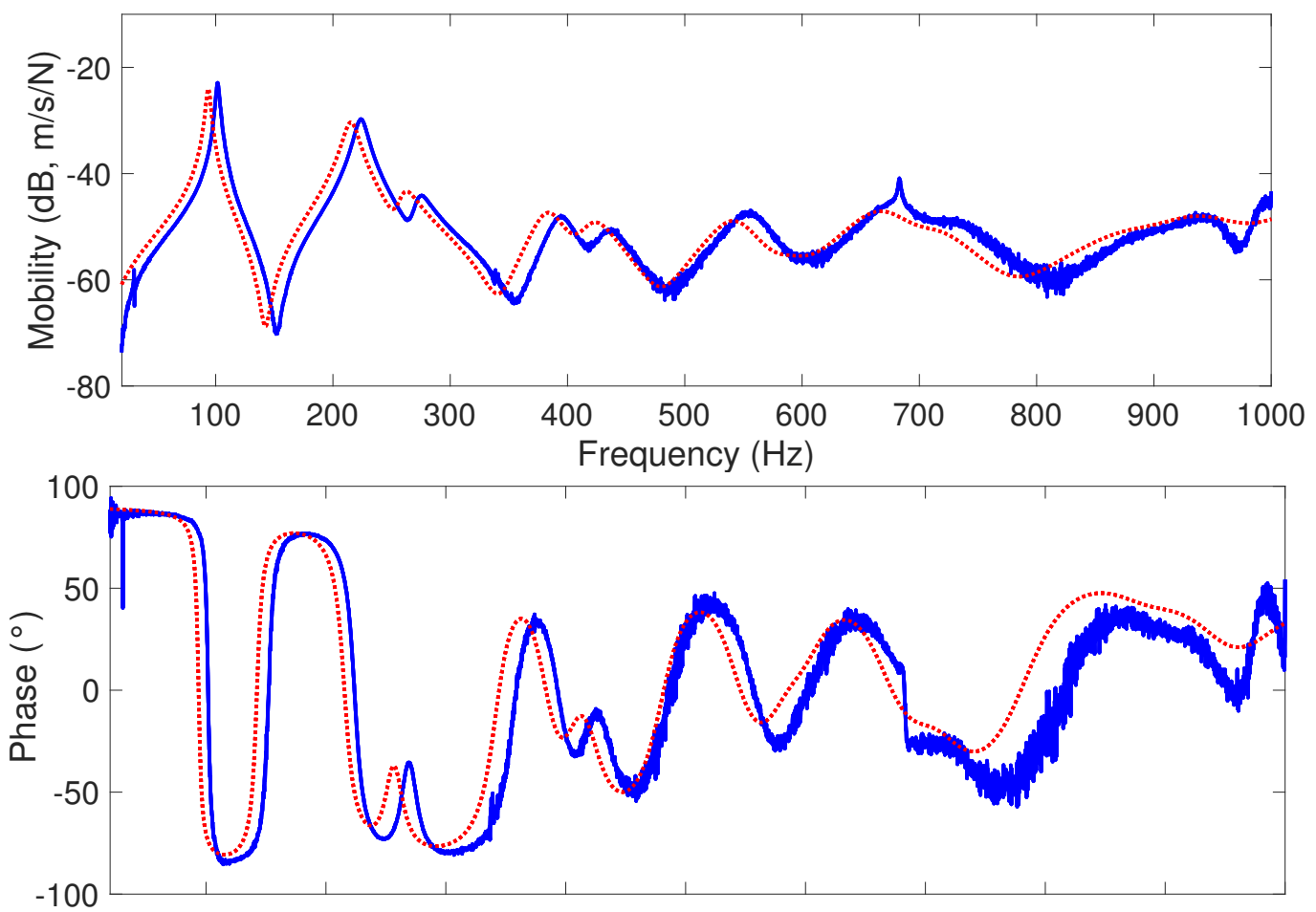

Fig. 15: Experimental (-) and numerical $(\cdots)$ mobilities for the panel with viscoelastic patches.

Then, Fig. 16 compares the estimated damping ratios for the first six vibration modes extracted from the numerical and experimental frequency responses through a Rational Fraction Polynomial method [23]. The presence of a flexural mode of the experimental frame around $685 \mathrm{~Hz}$ does not allow a proper estimation of the modal parameters between $600 \mathrm{~Hz}$ and $800 \mathrm{~Hz}$. Yet, results indicate that the proposed finite element model is able to predict rather accurately the damped response of the panel with viscoelastic patches. The slightly overestimated damping ratios in Fig. 16, along with the small frequency shift observed in Fig. 15 could be explained by the characterization of the viscoelastic material by DMA measurements. Despite this, the model is considered as validated and is used in the following sections to evaluate the damping performance of viscoelastic patches under different configurations.

\subsubsection{Influence of the viscoelastic layer thickness}

In order to study the influence of viscoelastic layer thickness on the dynamic response of the sandwich plate, several finite element calculations are carried out considering a thickness varying from $100 \mu \mathrm{m}$ to $300 \mu \mathrm{m}$. The damping ratios 


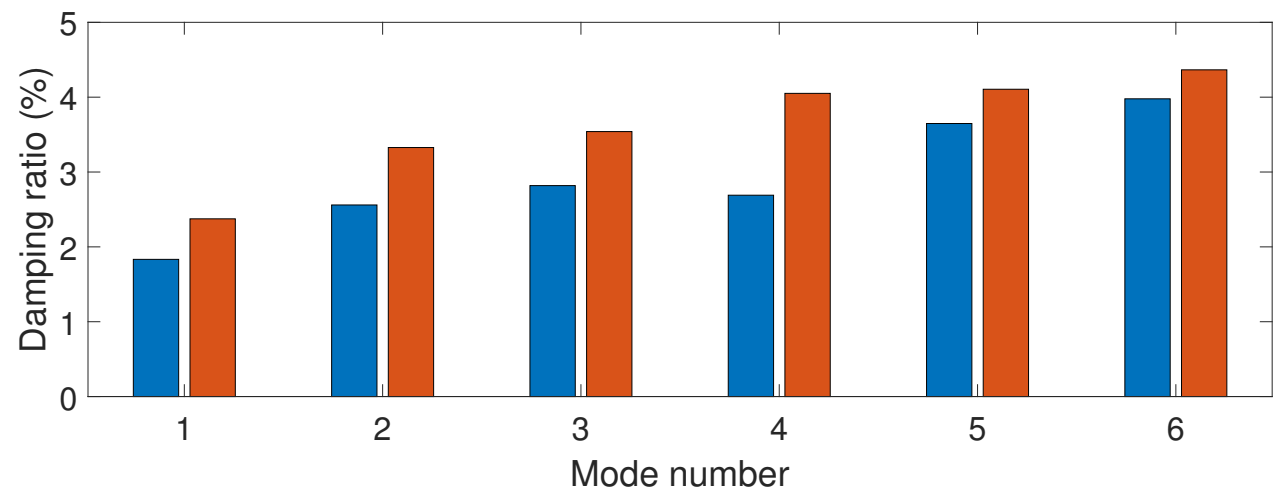

Fig. 16: Damping ratios for the experimental (left bars) and numerical (right bars) responses of the aluminum panel with viscoelastic patches.

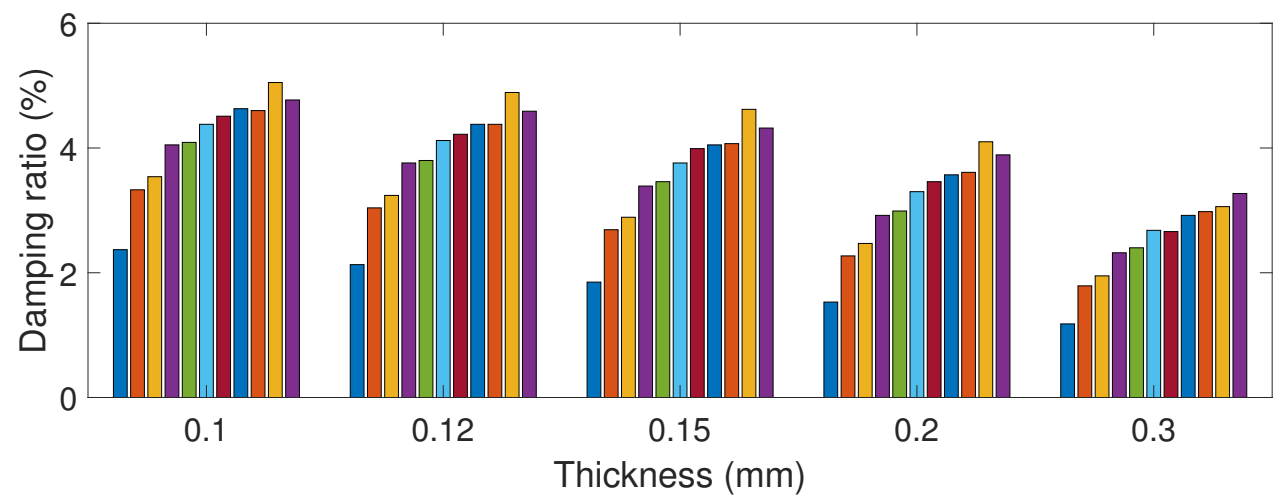

Fig. 17: Damping ratios for the first eleven modes of the panel with viscoelastic patches with varying thickness of the viscoelastic layer.

of the first eleven modes of the sandwich plate are estimated from the computed frequency responses. Figure 17 shows that the thinner the layer of viscoelastic material, the higher the damping ratios. According to the parametric study carried out in [26], there should be an optimal viscoelastic layer thickness, which from Fig. 17 appears to be less than $100 \mu \mathrm{m}$. In practice, such thin viscoelastic layers are difficult to achieve.

\subsubsection{Influence of the constraining layer thickness}

In order to study the influence of constraining layer thickness on the dynamic response of the sandwich plate, several finite element calculations are carried out considering a thickness varying from $0.52 \mathrm{~mm}$ to $2.60 \mathrm{~mm}$. Figure 18 shows that the thicker the constraining layer, the higher the damping ratios. This is also in accordance with the parametric study carried out in [26]. The ratio between the viscoelastic layer's thickness and that of the constraining layer is actually a good indicator of the performance of a passive damping treatment with viscoelastic patches.

\subsubsection{Influence of the number of patches}

The choice of 42 viscoelastic patches periodically distributed on the plate was dictated by the experimental comparison carried out between both passive damping treatments. Viscoelastic patches are classically applied as a single patch on the vibrating structure. Figure 19 compares the damping ratios extracted from the frequency responses computed with 42 patches and with one patch of equivalent surface, centered on the panel as seen in Fig. 13c. One can observe that the single patch solution leads to better damping performance. It should be noted that an optimum placement of the viscoelastic patches may be obtained through multi-objective optimization [27]. However, from a practical and industrial point of view, the application of a single constrained viscoelastic patch remains a common practice. Therefore, the single patch configuration is considered in the next section. 


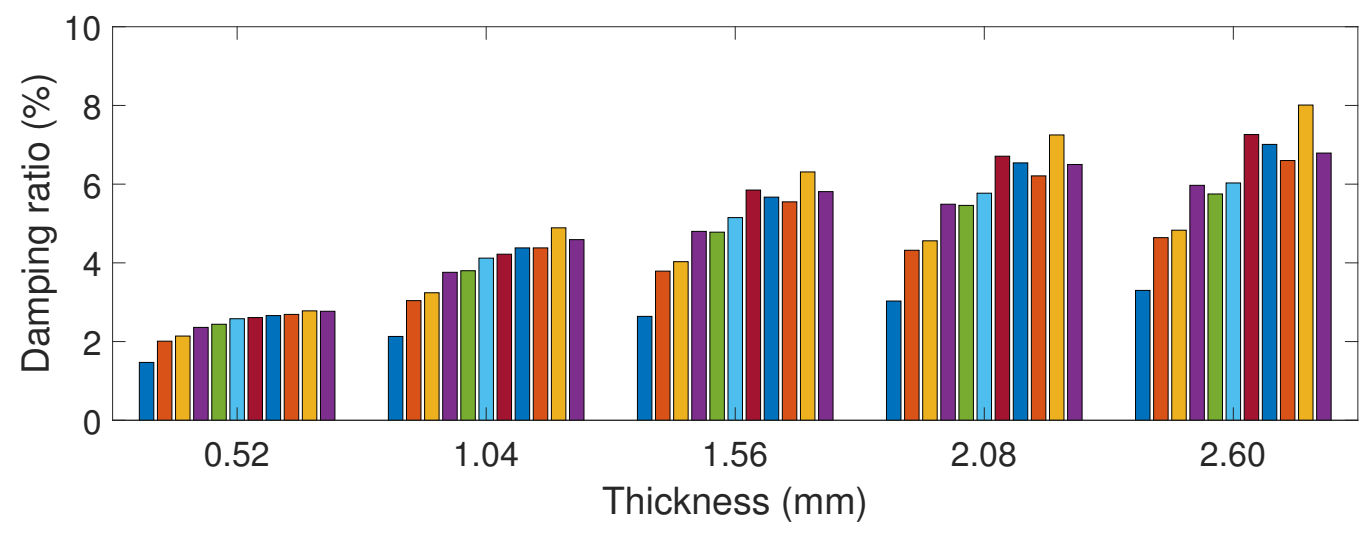

Fig. 18: Damping ratios for the first ten modes of the panel with viscoelastic patches with varying thickness of the constraining layer.

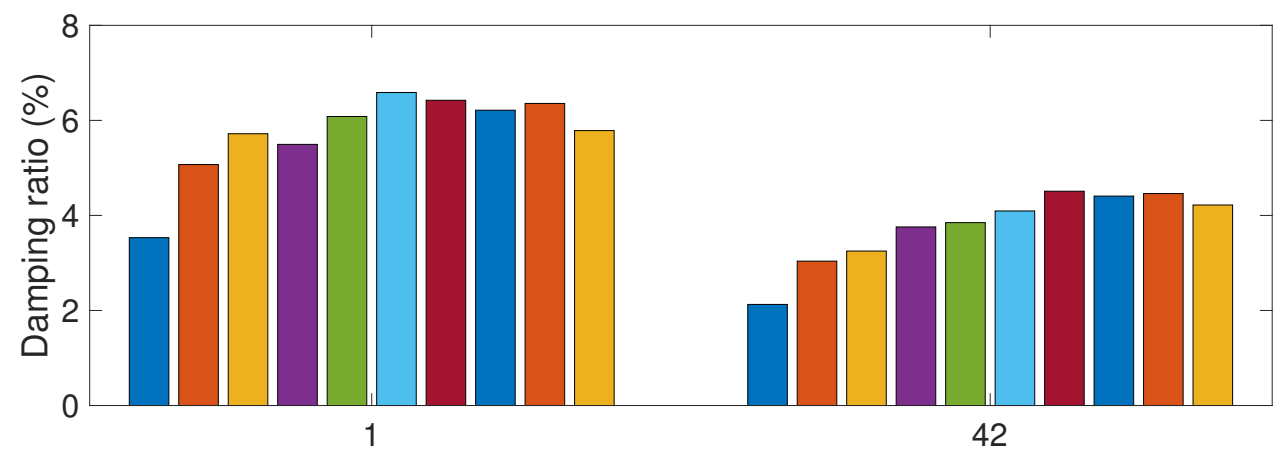

Number of patches

Fig. 19: Damping ratios for the first ten modes of the aluminum panel with 42 viscoelastic patches and one single patch of equivalent surface.

\section{Comparison of the damping treatments}

Weight and dimensions are classical constraints in the design of a damping treatment. Therefore, this section is dedicated to the objective comparison of damping performance through the numerical study of two configurations: equal-mass and equal-thickness.

\subsection{Equal-mass configuration}

In order to obtain an equal-mass configuration for the viscoelastic and the piezoelectric treatments (the mass of the electrical circuit is not taken into account), the thickness of the piezoelectric patches is modified in the numerical model: $0.23 \mathrm{~mm}$ instead of $0.5 \mathrm{~mm}$. The viscoelastic patches are still composed of a $120 \mu \mathrm{m}$ thick viscoelastic layer and a $1.04 \mathrm{~mm}$ thick constraining layer. In this way, both the single viscoelastic patch in Fig. $13 \mathrm{c}$ and the 42 piezoelectric patches have a total mass of $182 \mathrm{~g}$ (to be compared to the mass of the bare aluminum panel which is $1270 \mathrm{~g}$ ). For the piezoelectric treatment, the electrical network has been tuned in order to maximize the damping ratio of the second mode of the plate. The resulting damping ratios and frequency response functions for both panels are compared in Figs. 20 and 21. Results show that up to the fifth mode which is around $500 \mathrm{~Hz}$, the damping ratios are in the same order of magnitude for both damping treatments. Some piezoelectric damping ratios offer greatest values but note that in the case of forced vibrations, the frequency response function of the piezoelectric plate shows higher amplitude because all plate modes are doubled when coupled to the multi-resonant network. In a situation maximizing the damping ratio (for mode 2 here), the summed contributions of two modes with equal natural frequencies and equal damping ratios generate vibration amplitude that is twice that observed for a single mode with similar damping.

Moreover, considering the second half of the frequency range (modes 6 to 11), Fig. 20 shows that the viscoelastic panel offers damping ratios above $6 \%$, which is not the case with the piezoelectric panel that encounters a serious drop in added damping. This has a direct impact on the forced response in Fig. 21, where the difference between the 


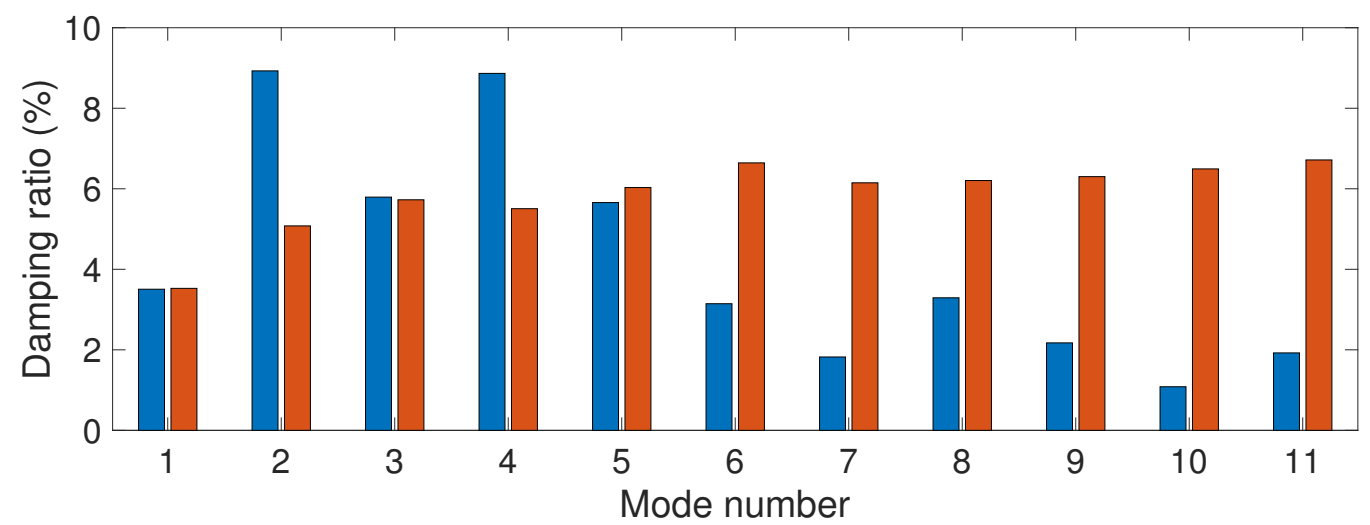

Fig. 20: Damping ratios for equal-mass configuration: with piezoelectric treatment (left bars) and with viscoelastic treatment (right bars).

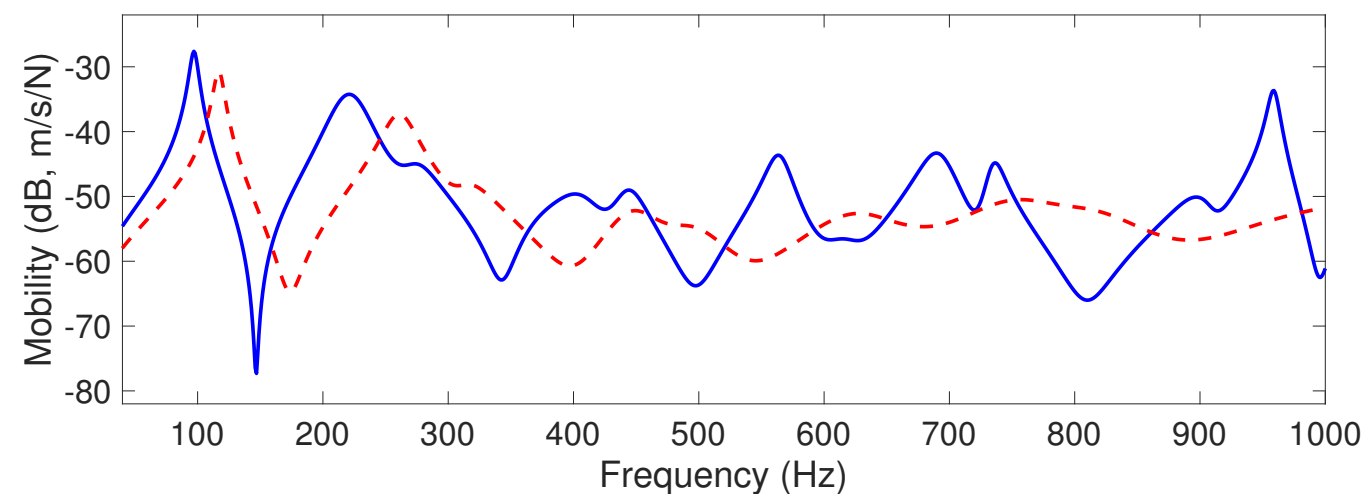

Fig. 21: Comparison of frequency response functions in equal-mass configuration: with piezoelectric treatment ( - ) and with viscoelastic treatment $(--)$.

two damping treatments can be easily observed. Such as seen in Fig. 9, a different network tuning could offer larger piezoelectric damping for the highest modes but, with the present network, it would necessary decrease damping of the first ones. In the end, if added mass is the primary constraint, piezoelectric network damping is probably not the best choice for a fully passive treatment because constrained viscoelastic layers can provide similar damping ratios with a lower cost and easier integrability.

\subsection{Equal-thickness configuration}

The equal-thickness configuration is obtained by modifying the thickness of the viscoelastic patches in the numerical model: $120 \mu \mathrm{m}$ for the viscoelastic layer (thinnest layer currently available) and $0.38 \mathrm{~mm}$ for the constraining layer. In this way, both viscoelastic and piezoelectric patches have a total thickness of $0.5 \mathrm{~mm}$. In this configuration, the added mass of the piezoelectric patches is $400 \mathrm{~g}$ while that of the viscoelastic patches is $74 \mathrm{~g}$.

The computed damping ratios and frequency response functions for both panels are compared in Figs. 22 and 23. This equal-thickness configuration identifies the piezoelectric solution as the most efficient damping technique over the whole frequency range. Damping ratios obtained from the piezoelectric network are significantly larger for the first modes. The difference is lower for the highest modes but a different electrical tuning as proposed in Figs. 9 and 10 would easily increase damping at higher frequencies. Note that the damping ratios for a smaller viscoelastic layer over backing layer ratio would be slightly larger, but the conclusions would remain the same with regard to the comparison with the piezoelectric damping solution.

To go further into the comparison of both treatments, it is possible to model a wide band input spectrum and quantify its effect in terms of root-mean-square (RMS) displacement. As an example, we choose a unitary power spectral density (PSD) input so that the RMS displacement can be directly calculated from the integral of the square of the compliance frequency response function. Table 5 shows the results for the experimental, equal-mass and equal-thickness configurations. The previous conclusion remain valid although the two treatments were not optimized 


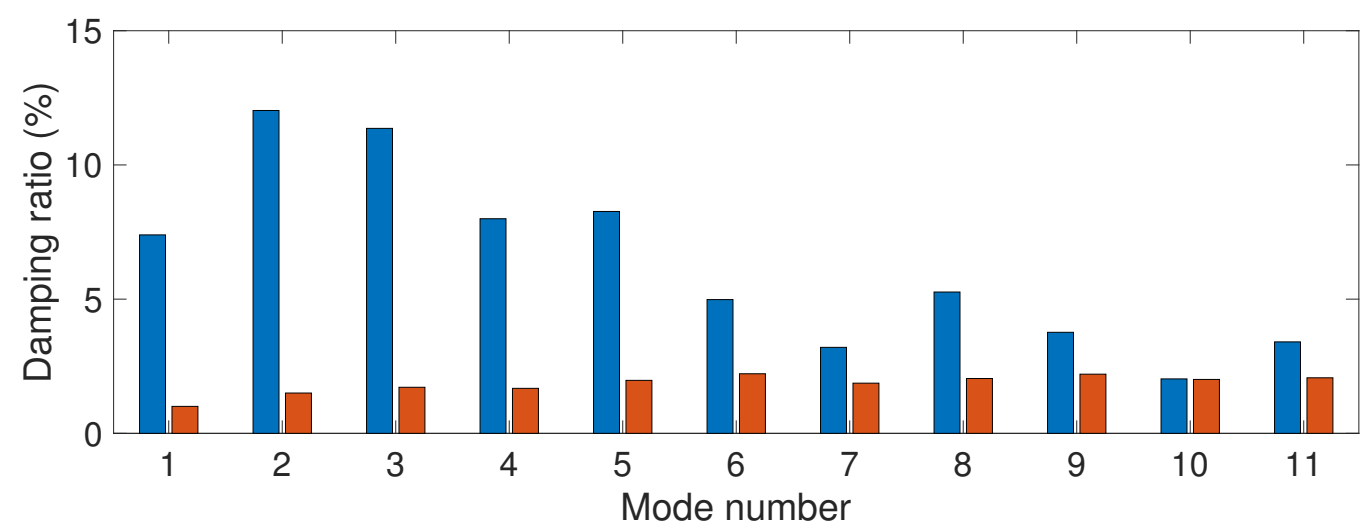

Fig. 22: Damping ratios for equal-thickness configuration: with piezoelectric treatment (left bars) and with viscoelastic treatment (right bars).

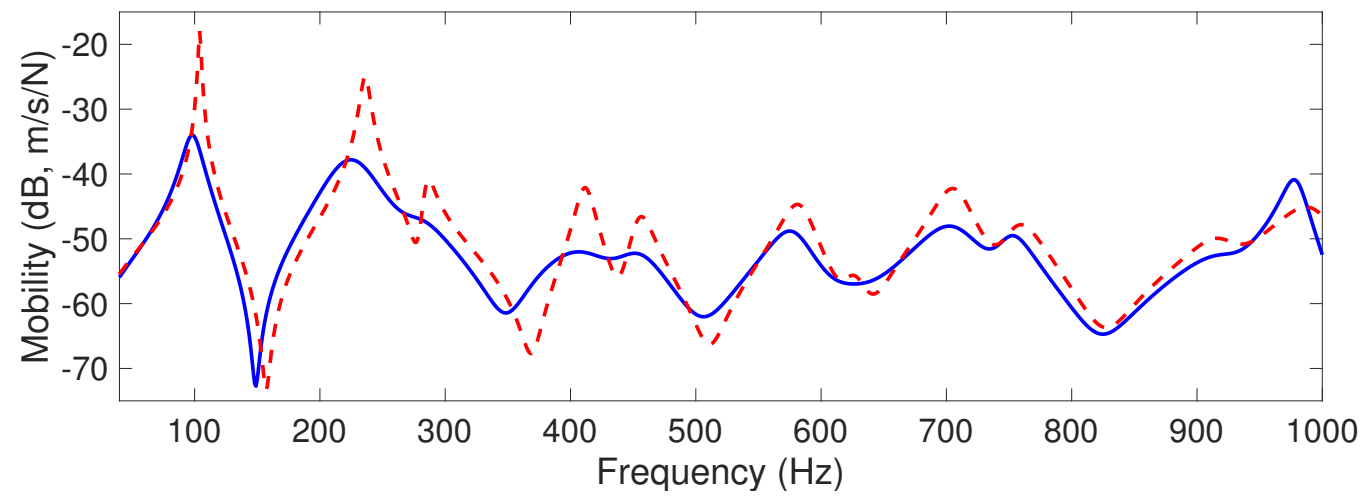

Fig. 23: Comparison of frequency response functions in equal-thickness configuration: with piezoelectric treatment $(-)$ and with viscoelastic treatment (--).

\begin{tabular}{cccc}
\hline Configuration & Experimental & Equal-mass & Equal-thickness \\
\hline Bare plate & 2.67 & - & - \\
Piezoelectric & 0.56 & 1.05 & 0.71 \\
Viscoelastic & 1.22 & 0.68 & 1.66 \\
\hline \hline
\end{tabular}

Table 5: RMS displacements (in $\mathrm{mm}$ ) for a unitary PSD input from $40 \mathrm{~Hz}$ to $1 \mathrm{kHz}$.

for this specific RMS objective. See for instance the piezoelectric planel that offer a lower RMS displacement in the experimental case compared to the equal-mass configuration obtained after maximization of the damping ratio on mode 2.

Finally, the results of this comparative study show that design constraints have a strong impact on the relative damping performance of viscoelastic and piezoelectric patches. For any industrial application, one has to precisely define all the specifications before rejecting one of the two solutions. Ideally, both damping treatments needs to be modelled for each industrial case because the present panel setup only provides guidelines obtained from a specific geometry.

\section{Conclusion}

The goal of this work was to compare the damping performance of two passive treatments based on constrained viscoelastic layers and piezoelectric patches connected to a multi-resonant electrical network. To this end, experimental and numerical tests have been carried out on an aluminum panel, equipped with viscoelastic or piezoelectric patches. Numerical models of both panels have been experimentally validated, which enables the numerical study of two configurations: equal-mass and equal-thickness configurations. 
The main conclusions of this work are :

- For an equal-mass configuration, both damping treatments can lead to damping ratios of similar orders of magnitude. However, considering the constraints related to broadband electrical tuning and integration of the electrical network, a viscoelastic treatment would generally be preferred as a lower-cost solution with high integrability.

- For an equal-thickness configuration, piezoelectric damping is preferable because multi-resonant piezoelectric networks can provide significantly higher damping compared to constrained viscoelastic layers. In this configuration, resistive piezoelectric networks could be another alternative with easier integrability at the expense of vibration mitigation performance.

It should be noted that this study was carried out on a specific structure, a simply supported panel, and the conclusions may differ depending on the intended application. The impact of the design constraints, such as cost, durability, integrability and temperature, should be further investigated for a fully objective comparison of the damping performance of both passive treatments. This yet requires a precise definition of the input specifications, which strongly depends on the considered industrial case.

\section{Authors' Contributions}

B. Lossouarn and L. Rouleau improved available finite element models to extract, analyze and present the results shown in this manuscript. R. Darleux conducted the experiments and developed the model of the panel coupled to the electrical network after J.-F. Deü provided the main structure of the piezoelectric finite element code. All authors reviewed the final manuscript.

\section{Acknowledgements}

We gratefully acknowledge Safran Aircraft Engines for their contribution to the realization of the panel with viscoelastic patches.

\section{References}

[1] A. Nashif, D. Jones, and J. Henderson. Vibration damping. John Wiley \& Sons, 1985.

[2] M. Rao. Recent application of viscoelastic admping for noise control in automobiles and commercial planes. Journal of Sound and Vibration, 262:457-474, 2003.

[3] N. W. Hagood and A. von Flotow. Damping of structural vibrations with piezoelectric materials and passive electrical networks. Journal of sound and vibration, 146(2):243-268, 1991.

[4] A. Preumont, B. De Marneffe, A. Deraemaeker, and F. Bossens. The damping of a truss structure with a piezoelectric transducer. Computers \& structures, 86(3-5):227-239, 2008.

[5] K. Yamada, H. Matsuhisa, H. Utsuno, and K. Sawada. Optimum tuning of series and parallel LR circuits for passive vibration suppression using piezoelectric elements. Journal of sound and vibration, 329(24):5036-5057, 2010.

[6] J. Høgsberg and S. Krenk. Balanced calibration of resonant shunt circuits for piezoelectric vibration control. Journal of Intelligent Material Systems and Structures, 23(17):1937-1948, 2012.

[7] O. Thomas, J. Ducarne, and J.-F. Deü. Performance of piezoelectric shunts for vibration reduction. Smart Materials and Structures, 21(1):015008, 2012

[8] P. Gardonio and D. Casagrande. Shunted piezoelectric patch vibration absorber on two-dimensional thin structures: Tuning considerations. Journal of Sound and vibration, 395:26-47, 2017.

[9] C. Maurini, F. Dell'Isola, and D. Del Vescovo. Comparison of piezoelectronic networks acting as distributed vibration absorbers. Mechanical Systems and Signal Processing, 18(5):1243-1271, 2004.

[10] I. Giorgio, A. Culla, and D. Del Vescovo. Multimode vibration control using several piezoelectric transducers shunted with a multiterminal network. Archive of Applied Mechanics, 79(9):859-879, 2009. 
[11] M. Berardengo, S. Manzoni, and A. Conti. Multi-mode passive piezoelectric shunt damping by means of matrix inequalities. Journal of Sound and Vibration, 405:287-305, 2017.

[12] G. Raze, A. Paknejad, G. Zhao, C. Collette, and G. Kerschen. Multimodal vibration damping using a simplified current blocking shunt circuit. Journal of Intelligent Material Systems and Structures, 31(14):1731-1747, 2020.

[13] R. Darleux, B. Lossouarn, and J.-F. Deü. Broadband vibration damping of non-periodic plates by piezoelectric coupling to their electrical analogues. Smart Materials and Structures, 29(5):054001, 2020.

[14] L. Rouleau, R. Pirk, B. Pluymers, and W. Desmet. Characterization and modeling of the viscoelastic behavior of a self-adhesive rubber using dynamic mechanical analysis tests. Journal of Aerospace Technology and Management, 7(2):200-208, 2015.

[15] C. Johnson. Design of passive damping system. Journal of Vibration and Acoustics, 117:171-176, 1995.

[16] L. Rouleau, B. Lossouarn, R. Darleux, and J.-F. Deü. Comparison of damping performances of constrained viscoelastic layers and passive piezoelectric networks. In 48th International Congress and Exposition on Noise Control Engineering, INTERNOISE 2019, Madrid, Spain, June 2019.

[17] D. Oshmarin, N. lurlova, N. Sevodina, and M. Iurlov. A comparative analysis of two approaches to damping of vibrations. Procedia Structural Integrity, 28:1438-1448, 2020.

[18] O. Robin, J.-D. Chazot, R. Boulandet, A. Berry, and N. Atalla. A plane and thin panel with representative simply supported boundary conditions for laboratory vibroacoustic tests. Acta Acustica united with Acustica, 102:170-182, 2016.

[19] O. Robin, A. Berry, N. Atalla, M. Aucejo, B. Lossouarn, et al. Setting up plane and thin panels with representative simply supported boundary conditions: comparative results and applications in three laboratories. In Proceedings of Inter-noise 2018, Chicago, Illinois, August 2018.

[20] M. A. Trindade and A. Benjeddou. Effective electromechanical coupling coefficients of piezoelectric adaptive structures: critical evaluation and optimization. Mechanics of Advanced Materials and Structures, 16(3):210-223, 2009.

[21] B. Lossouarn, M. Aucejo, J.-F. Deü, and K. Kunefare. Design of a passive electrical analogue for piezoelectric damping of a plate. Journal of Intelligent Material Systems and Structures, 29(7):1301-1314, 2018.

[22] O. Thomas, J.-F. Deü, and J. Ducarne. Vibrations of an elastic structure with shunted piezoelectric patches: efficient finite element formulation and electromechanical coupling coefficients. International journal for numerical methods in engineering, 80 (2):235-268, 2009.

[23] M. H. Richardson and D. L. Formenti. Parameter estimation from frequency response measurements using rational fraction polynomials. In Proceedings of the 1st international modal analysis conference, volume 1, pages 167-186. Union College Schenectady, NY, 1982.

[24] S. Adhikari. Damping models for structural vibration. PhD thesis, Cambridge University, 2000.

[25] S. Erlicher and P. Argoul. Modal identification of linear non-proportionally damped systems by wavelet transform. Mechanical Systems and signal processing, 21(3):1386-1421, 2007.

[26] B. R. Sher and R. Moreira. Dimensionless analysis of constrained damping treatments. Composite Structures, 99:241-254, 2013.

[27] J. Madeira, A. Araújo, C. Mota Soares, and C. Mota Soares. Multiobjective optimization for vibration reduction of composite plate structures using constrained layer damping. Computers and Structures, 232:105810, 2020. 